\title{
Array-Based Vapor Sensing Using Chemically Sensitive, Polymer Composite Resistors
}

\author{
Mark C. Lonergan, ${ }^{*}$ Michael S. Freund, ${ }^{* *}$ Erik J. Severin, Brett J. Doleman, Robert H. Grubbs, \\ and Nathan S. Lewis ${ }^{\dagger}$ \\ Division of Chemistry and Chemical Engineering \\ California Institute of Technology \\ 1200 E. California Blvd. \\ Pasadena, CA, 91125 \\ (818) 395-6335 \\ nslewis@cco.caltech.edu
}

\section{ABSTRACT}

We describe herein the construction of simple, low-power, broadly responsive vapor sensors. Insulating polymer-conductor composites have been shown to swell reversibly upon exposure to vapors. Thin films of polymer composites have been deposited across two metallic leads, with swelling-induced resistance changes of the films signaling the presence of vapors. To identify and classify vapors, arrays of such vapor-sensing elements have been constructed, with each element containing either carbon black or poly(pyrrole) as the conducting phase mixed with one of several different organic polymers as the insulating phase. A convenient chemical polymerization of poly(pyrrole) which allows a high degree of processibility is also described. The differing gas-solid partition coefficients for the various polymers of the sensor array produce a pattern of resistance changes that can be used to classify vapors and vapor mixtures. This type of sensor array has been shown to resolve common organic solvents, including molecules of different classes (such as aromatics from alcohols) as well as those within a particular class (such as benzene from toluene and methanol from ethanol). The response of an individual composite to varying concentrations
\end{abstract}

of solvent is shown to be consistent with the predictions of percolation theory. Accordingly, significant increases in the signals of array elements have been observed for carbon black-polymer composites that were operated near their percolation thresholds.

*Present address: Department of Chemistry, University of Oregon-Eugene

** Present address: Department of Chemistry, Lehigh University, 6 East Packer Avenue, Bethlehem, PA 18015-3172

${ }^{\dagger}$ Author to whom correspondence should be addressed.

\section{INTRODUCTION}

Conventional approaches to chemical sensors have traditionally made use of a "lock-andkey" design, wherein a specific receptor is synthesized in order to bind strongly and highly selectively to the analyte of interest. A related approach involves exploiting a general physicochemical effect selectively toward a single analyte, such as the use of the ionic effect in the construction of a $\mathrm{pH}$ electrode. With both of these approaches, selectivity is achieved through precise chemical design of the receptor site. Such approaches are appropriate when a specific target compound is 
to be identified in the presence of controlled backgrounds and interferences. However, this type of approach requires the synthesis of a separate, highly selective sensor for each analyte to be detected. In addition, this type of approach is not particularly useful for analyzing, classifying, or assigning human value judgments to the composition of complex vapor mixtures such as perfumes, beers, foods, mixtures of solvents, etc.

An alternative approach to chemical sensing is closer conceptually to a design widely proposed for the mammalian sense of olfaction. ${ }^{1,2}$ In such an approach, the strict "lock-and-key" design criterion of traditional sensing devices is abandoned. Instead, in this alternative sensor architecture, an array of incrementally different sensors is used, with every element in the sensor array chosen to respond to a number of different chemicals or classes of chemicals. ${ }^{3-6}$ The elements of such an array should contain as much chemical diversity as possible, so that the array responds to the largest possible cross-section of analytes. Although in this design identification of an analyte cannot be accomplished from the response of a single sensor element, a distinct pattern of responses produced over the collection of sensors in the array could provide a fingerprint that would allow classification and identification of the analyte. The advantage of this approach is that it can yield responses to a variety of different analytes, including those for which the array was not originally designed. In addition, the broadly responsive sensors need not incorporate synthetically challenging, customdesigned, "lock-and-key" receptor sites in order to generate a response to an analyte. Also, an array of sensors naturally performs an integration to yield a unique signal for complex but distinctive odors (e.g., cheeses, beers, etc.) without requiring that the mixture be broken down into its individual components prior to, or during, the analysis.
We describe herein a simple, broadly responsive sensor array, based on polymerconductor composites ${ }^{7-9}$ and demonstrate that this array can classify, detect, and quantify various test vapors and vapor mixtures. The individual sensor elements are constructed from films consisting of carbon black or poly(pyrrole) dispersed into insulating organic polymers. The carbon black or poly(pyrrole) endows electrical conductivity to the films, whereas the different organic polymers are the source of chemical diversity between elements in the sensor array. Swelling of the polymer upon exposure to a vapor increases the resistance of the film, thereby providing an extraordinarily simple means for monitoring the presence of a vapor. ${ }^{7,10-16}$ Since different polymer compositions are present on each sensor element, an array of elements responds to a wide variety of vapors (or complex mixtures of vapors) in a distinctive, identifiable fashion (Figure 1). The electrical resistance signals that are output from the array can be readily integrated into software- or hardwarebased neural network processors, allowing for an integration of sensing and analysis functions into a compact, low-power, simple vapor sensor.

Array-based vapor sensing has been demonstrated previously in several systems, including those using surface acoustic wave devices, ${ }^{17-20}$ tin oxide sensors, ${ }^{21-23}$ and conducting organic polymers. ${ }^{24-26}$ In general, desirable design criteria for the elements of such an array are as follows: (1) they should readily transduce environmental information into an easily monitored signal, using a minimum of hardware and energy; (2) they should exhibit reversible, reproducible responses with a minimum of baseline drift; (3) they should be broadly tunable to respond in a predictable manner to a wide range of chemical species and concentrations; (4) they should be easily fabricated, preferably from inexpensive, commercially-available materials using well-established techniques; (5) they 
should permit miniaturization to facilitate the construction of compact sensors with a large number of elements; and (6) they should be robust and stable in many different environments. $\mathrm{SnO}_{2}$ gas sensors ${ }^{21-23}$ are among the most well-established sensing elements, and several commercial "electronic noses" have been based on $\mathrm{SnO}_{2}$ arrays. ${ }^{6}$ Although such arrays yield diagnostic responses for several gases, the incomplete understanding of catalytic processes at the doped $\mathrm{SnO}_{2}$ surface makes chemical control of the response properties, and thus deliberate introduction of desired chemical diversity into the array, difficult to accomplish. Surface acoustic wave (SAW) devices are extremely sensitive to the presence of vapors, but involve somewhat sophisticated electronics to sustain surface Rayleigh waves in the piezoceramic crystals. Chemical diversity in a SAW array can be readily attained by coating the SAW crystals with different polymer films having differing gas-solid partition coefficients towards a vapor of interest. To our knowledge, primarily because of the electronic complexity involved in a SAW device and the resulting engineering challenges associated with micromanufacturing large numbers of such systems into an integrated system, the largest SAW array reported to date contains approximately 12 sensor elements. ${ }^{17-20}$ In contrast, over 1000 receptor genes have recently been discovered in the mammalian olfactory system, and it is therefore estimated that the dimensionality of smell in humans is approximately $10^{3} .{ }^{27}$ There is thus great intellectual interest in constructing sensor arrays that have large numbers of chemically distinct sensor elements so that a large number of diverse sensing tasks can be accommodated within one array structure, and to investigate fundamentally the behavior of systems that are functionally, if not structurally, analogous to the mammalian olfactory response. Conducting organic polymers have also been used to form sensor arrays, ${ }^{24,25}$ and commercial "electronic nose" devices have recently been announced using pure poly(pyrroles) (no insulating polymer matrix present) ${ }^{28}$ However, since there are only a few classes of stable conducting polymers, and since to date the conducting polymers have been synthesized electrochemically to yield insoluble, intractable materials, additional variation in the array elements has been largely confined to changes in the counterion of the polymer or to the more synthetically challenging task of varying the substituents on the polymer backbone.

The scope of conducting polymer-based sensors has been broadened through the use of a set of polymer blends that possess a common conducting element, poly(pyrrole), for signal transduction, and a variety of insulating, swellable, organic polymers to achieve chemical diversity in the array. ${ }^{26}$ Our approach has been to prepare processable thin films of electrically conducting organic polymers as the individual sensor elements. Use of processable films has allowed deliberate control over the chemical properties of the resulting conducting polymer coatings. Specifically, we have utilized the chemical polymerization of pyrrole under controlled conditions to produce thin conducting films on non-conducting substrates.

These devices have been shown to function quite well, but the long-term stability of poly (pyrrole) is of concern for practical use of such systems. Therefore, the use of carbon black as the conductor has been explored. The advantages of using carbon black is that it is a very stable species. Furthermore, chemical diversity in the sensor array can be simply obtained through the use of the organic polymers functioning as the insulating phase of the carbon black composites. Individual carbon black composites have been widely explored as humidity sensors $7,11-13$ and, to a somewhat lesser extent, as sensors for organic vapors or liquids such as gasoline. ${ }^{10,14-16}$ 
In this paper, we demonstrate the feasibility of using conductor-organic polymer composite films that have distinctly different electrical resistance responses to various vapors, and have obtained diagnostic array signal patterns in response to a series of test odorants. We also demonstrate, in the case of carbon black, how the properties of these individual sensors can be tuned through variation in the insulating polymer and conductor content of the composite films.

\section{EXPERIMENTAL}

\section{A. Materials}

The carbon black used in the composites was Black Pearls 2000 (BP2000), a furnace black material that was generously donated by Cabot Co. (Billerica, MA). The insulating polymers used in the carbon black and poly(pyrrole) composite sensors (Tables $1 \mathrm{a}$ and $\mathrm{lb}$ ) were purchased from Polysciences Inc. or Aldrich Chemical Co. and were used as received. The solvents used in this study were all were reagent grade and were used as received from EM Scientific. The pyrrole and phosphomolybdic acid were obtained from Aldrich.

\section{B.Fabrication of Carbon Black Composite Sensors}

Ceramic capacitors ( $22 \mathrm{nF}$, approx. $2 \times 4 \times 4$ $\mathrm{mm}$ ) from Kemet Electronics (Greenville, SC) were found to provide a very convenient electrical contact and physical support for the composite films of each sensor. First, the interdigitated electrodes inside the capacitor were exposed by using progressively finer grades of sandpaper and polishing paste to remove the top of the capacitor. During this process, the bulk of the material was removed with diamond-impregnated sanding paper on a sanding belt. The path of the grinding paper or paste was parallel to the interdigitated electrodes to avoid shorting the capacitor.
Following the diamond paper treatment, the capacitors were sanded on a disk sander using 3M Tri-M-ite Fre-Cut, Open Coat, 360 grit paper. The tops of the capacitors were then polished on a 48-1581-BXXR polishing wheel (Buehler LTD, Lake Bluff, IL) using $0.3 \mu \mathrm{m}$ diameter Buehler $\alpha$-alumina micropolish grit. After the polishing step, the capacitors were sonicated in acetone or 2-propanol for 5-10 min to remove any residual alumina. All capacitors that were used as sensors had an initial resistance after polishing of greater than $10 \mathrm{M} \Omega$ (greater than the upper measurement limit of our ohmmeter).

Individual sensor elements were prepared by a single dip of the polished, cleaved capacitors into $10 \mathrm{~mL}$ solutions that contained $80 \mathrm{mg}$ of dissolved polymer and $20 \mathrm{mg}$ of suspended carbon black. After removal from the solution, any excess liquid was shaken off or blotted off, and the film was then dried in air prior to use. The solvent was generally THF, but benzene was the solvent for composites prepared from poly(ethylene - co - vinyl acetate) and poly(ethylene oxide), and dichloromethane was the solvent for composites made from poly(caprolactone). Prior to immersion of the capacitor, the solutions were sonicated for 5-10 min to aid in the suspension of the carbon black particles.

Some studies were also performed using glass substrates instead of the ceramic capacitors described above. To prepare the glass substrates, two parallel bands of gold, 0.5-1.0 $\mu \mathrm{m}$ thick and separated by $5 \mathrm{~mm}$, were deposited onto conventional $7.5 \mathrm{~cm} \times 2.5 \mathrm{~cm}$ glass slides. The slides were then cut into strips to produce $0.7 \mathrm{~cm} \times 2.5 \mathrm{~cm}$ pieces of glass, with each strip of glass having one pair of Au leads spaced $5 \mathrm{~mm}$ apart.

When glass substrates were used, a slightly different procedure was utilized to form the composite films. Appropriate aliquots (4-15 
$\mathrm{mL}$ in volume depending on the final desired polymer concentration) of stock polymer solutions (6 $\mathrm{mg} \mathrm{mL}^{-1}$ ) were diluted to a volume of $15 \mathrm{~mL}$ using the same solvent as that in the stock solution. Carbon black was added to this solution until the total mass of polymer and carbon black was $100 \mathrm{mg}$. Two glass substrates were then dipped back to back (the front being the side with the gold leads) into this solution. The slides were held with a forceps, dipped into the solution, and removed quickly $(0.1-0.5 \mathrm{~s})$. The slides were dipped several times with 5-10 $\mathrm{s}$ of air drying between dips. Studies using glass substrates were only performed with poly(ethylene - co - vinyl acetate) (PEVA, 82\% (w/ w) ethylene) or poly $(N$-vinylpyrrolidone) (PVP) carbon black composites. The PEVA slides were dipped 4 times, while the PVP slides were dipped 10 times into the fabrication solution.

At the lowest carbon black loadings studied, the composite films of PVP and PEVA appeared speckled under $40 \mathrm{X}$ magnification, with the largest carbon black aggregates being $\approx 30 \mu \mathrm{m}$ in length and irregular in shape. With increasing carbon black loading, the aggregate size increased until eventually the films appeared uniformly black under 40X magnification. Profilometry data obtained using a Dektak 3030 profilometer (Sloan Technology Corp., Santa Barbara, CA) on these films showed average film thicknesses ranging from $0.2-2 \mu \mathrm{m}$.

\section{C.Fabrication of Poly(pyrrole) Composite Sensors}

Poly(pyrrole) films used for conductivity, electrochemical, and optical measurements were prepared by injecting equal volumes of $\mathrm{N}_{2}$-purged solutions of pyrrole ( 1.50 mmoles in $4.0 \mathrm{ml}$ dry tetrahydrofuran) and phosphomolybdic acid $(0.75$ mmoles in $4.0 \mathrm{ml}$ tetrahydrofuran) into a $\mathrm{N}_{2}$-purged test tube. Once the two solutions were mixed, the yellow phosphomolybdic acid solution turned dark green, with no observable precipitation for several hours. This solution was used for film preparation within an hour of mixing.

Poly(pyrrole)-insulating polymer sensors were made by mixing two solutions, one of which contained 0.29 mmoles pyrrole in $5.0 \mathrm{ml}$ tetrahydrofuran, with the other containing 0.25 mmoles phosphomolybdic acid and $30 \mathrm{mg}$ of insulating polymer in $5.0 \mathrm{ml}$ of tetrahydrofuran. The mixture of these two solutions resulted in a w: $w$ ratio of pyrrole to insulating polymer of $2: 3$. The monomer insulating polymer - oxidant solutions were then used to dip coat interdigitated electrodes of the modified ceramic capacitors in order to provide a robust electrical contact to the polymerized organic films. After polymerization was complete, the film was insoluble and was rinsed with solvent (tetrahydrofuran or methanol) to remove residual phosphomolybdic acid and unreacted monomer. The sensors were then connected to a commercial bus strip, with the resistances of the various "chemiresistor" elements readily monitored by use of a multiplexing digital ohmmeter.

\section{Apparatus}

Standard glassware was used to construct a bubbler apparatus (to provide known partial pressures of various vapors) and a flow chamber to control the resulting gas stream. The bubblers were large test tubes $(30 \mathrm{~cm}$ long with a $3 \mathrm{~cm}$ inside diameter) equipped with exit sidearms. To provide a pathway for gas flow, a glass tube terminated by a coarse filter frit was inserted into a rubber stopper and then placed into the top of each bubbler. The carrier gas was compressed air from the general lab source, and was neither filtered nor dehumidified. The measurements were performed at room temperature, which was in the range $22 \pm 2{ }^{\circ} \mathrm{C}$ over the course of the experiments described herein. The carrier gas 
was introduced into the solvent through the porous ceramic frit, and the solvent-saturated gas mixture exited the bubbler via the sidearm of the glass tube. Saturation of the gas streams in our experimental apparatus was verified for the highest flow rates $\left(1.0 \mathrm{~L} \mathrm{~min}^{-1}\right)$ used in this work through measurement of the rate of mass loss of liquid in the bubbler, ${ }^{29}$ thus saturation conditions were assumed to have been obtained for the lower flow rates used in other experiments described in this work. The experimentally measured vapor pressures at the highest gas flow rate through the bubbler were within $2 \%$ of the values calculated from the literature ${ }^{30}$ for the measured temperatures of the solvent in the bubblers during the period of gas flow. The experimentally measured vapor pressures and corresponding solvent temperatures were as follows: acetone 176 Torr $\left(19^{\circ} \mathrm{C}\right)$; benzene 83 Torr $\left(22^{\circ} \mathrm{C}\right)$; chloroform 158 Torr $\left(20^{\circ} \mathrm{C}\right)$; ethanol 50 Torr $\left(22^{\circ} \mathrm{C}\right)$; ethyl acetate 82 Torr $\left(22^{\circ} \mathrm{C}\right)$; hexane 114 Torr $\left(19^{\circ} \mathrm{C}\right)$; methanol 102 Torr $\left(21^{\circ} \mathrm{C}\right)$; 2-propanol 37 Torr $\left(22^{\circ} \mathrm{C}\right)$; toluene 25 Torr $\left(23^{\circ} \mathrm{C}\right)$. The saturated vapor was carried out the sidearm of the bubbler, blended with a controlled background flow of pure carrier gas and then introduced into a sensing chamber. This chamber consisted of a glass tube $(22 \mathrm{~cm}$ long with a $2.6 \mathrm{~cm}$ inside diameter) to which inlet and outlet sidearms had been attached. The sensing elements were introduced into the chamber through a 24/40 taper ground glass opening attached at one end of the chamber. The chamber was then sealed with a groundglass stopper through which the electrical lead wires had been sealed. The gas flow rates were controlled with needle valves and stopcocks.

\section{E. Measurements}

To determine the response of the sensor elements to various vapors, the $\mathrm{dc}$ resistance of each sensor was determined as a function of time. Resistance measurements were performed using a simple two-point configuration. Sensors fabricated with the capacitor supports were plugged directly into a 40 pin bus strip that was then connected to a multiplexing ohmmeter via a ribbon cable. The resistances of the composite films on glass substrates were monitored similarly except that the gold leads on the glass slides were pressure-contacted with flat-jawed alligator clips.

Generally, resistance data were acquired using a Hydra 2620A Data Acquisition Unit (John Fluke Mfg. Co.) interfaced to a personal computer. All of the prepared samples had resistances less than the $10 \mathrm{M} \Omega$ limit of the Hydra 2620A. In some cases, however, swelling increased the sample resistance to above $10 \mathrm{M} \Omega$. In these cases, resistance measurements were performed using a Princeton Applied Research model 173 potentiostat or a Hewlett Packard model 6024 dc power supply (to apply a known potential) and a Keithley model 177 multimeter (to measure the resulting current across the resistive sensor element). In a few test cases, electrical resistance measurements were also made in a four-point configuration, and these data indicated that, in our experimental configuration, vapor-induced changes in contact resistance were minimal compared to the vapor-induced changes in the resistivity of the sensor films.

To initiate an experiment, the sensors were placed into the glass chamber and a background flow of compressed air was introduced until the resistance of the sensors stabilized. Solvent vapor streams of various concentrations and compositions were then passed over the sensors. The flow rates in the bubblers were controlled using flow meters obtained from Gilmont Instruments, Inc., with the lower and upper limits of the flow meters being either $0.2 \mathrm{~L} \mathrm{~min}^{-1}$ and $15.0 \mathrm{~L} \mathrm{~min}^{-1}$, $0.0010 \mathrm{~L} \mathrm{~min}^{-1}$ and $0.280 \mathrm{~L} \mathrm{~min}^{-1}, 0.0015 \mathrm{~L}$ $\min ^{-1}$ and $0.310 \mathrm{~L} \mathrm{~min}^{-1}$, or $0.0048 \mathrm{~L} \mathrm{~min}^{-1}$ 
and $0.673 \mathrm{~L} \mathrm{~min}^{-1}$ respectively. Analyte gas flows were kept low enough $\left(<1 \mathrm{~L} \mathrm{~min}^{-1}\right)$ to ensure that the vapor was saturated with solvent prior to dilution with the background gas.

\section{III.RESULTS AND DISCUSSION}

\section{A.Carbon Black Composite Sensors}

\section{Sensor Element Response Characteristics}

Figure 2 shows the resistance change of two carbon black-polymer composite films during repeated, periodic exposures to a test solvent vapor. The resistances of the films increased when the solvent vapor was present and then returned to their original baseline values after the vapor flow was discontinued. For example, Figure $2 \mathrm{a}$ shows data for fifteen sequential exposures of a PEVA (poly(ethylene - co - vinyl acetate, $82 \%$ (w / w) ethylene)carbon black composite film to $1.1 \mathrm{ppt}(\mathrm{ppt}=$ part per thousand $(\mathrm{v} / \mathrm{v})$ ) of benzene in air; Figure $2 b$ shows similar data for the exposure of a PVP (poly( $N$-vinylpyrrolidone))-carbon black composite film to $1.5 \mathrm{ppt}$ methanol in air. For the PEVA composite, resistance changes of $8.7 \pm 0.2 \mathrm{k} \Omega(0.77 \%$ of the baseline value) were observed for exposure to benzene vapor; for the PVP composite, resistance changes of $2.95 \pm 0.07 \mathrm{k} \Omega(2.28 \%$ of the baseline value) were observed for exposure to methanol. The form of the time response of these sensors were representative of all sensor elements studied in this work, with response times under these experimental conditions generally varying from $<2 \mathrm{~s}$ to $4 \mathrm{~s}$ for the film thicknesses used in this study $(2 \mathrm{~s}$ was the minimum time resolution of the multiplexing ohmmeter in this experiment). As can be seen from the data of Figure 2, the baseline resistance value drifted by approximately $<0.02 \%$ for the PEVA composite and $<0.15 \%$ for the PVP composite over a $20 \mathrm{~min}$ time period. These relative resistance changes and baseline drift rates were representative of the behavior of all sensor elements studied in this work under these experimental conditions.

It was of interest to examine the dependence of the signal response on the conductor/insulator ratio of a sensor element film. If the film composition could be manipulated so that solvent-induced swelling forced the film across its percolation threshold, very large resistance changes might be observed upon introduction of low concentrations of solvent vapor. Such a change should also produce a nonlinear signal vs. vapor concentration response, with the greatest sensitivity to vapor near the percolation threshold. Figure 3 displays such data for two PEVA-carbon black composite sensor elements, one fabricated from a $15 \%$ $(w / w)$ carbon black-PEVA mixture and the other from a $50 \%(\mathrm{w} / \mathrm{w})$ carbon black-PEVA mixture. The data in Figure 3 are semilog plots of the partial pressure dependence of the maximum relative differential resistance signals, $\Delta R_{\max , \infty} / R$, where $R$ is the baseline resistance of the film prior to exposure to the solvent and $\Delta R_{\max , \infty}$ is the maximum differential resistance signal that was observed in response to an extended exposure of the sensor to the specified partial pressure of solvent vapor. During exposure to benzene vapor, both sensor elements clearly displayed maximum relative differential resistance responses that were a function of the partial pressure, $P$, of the solvent. Below $P / P * \approx$ $0.81\left(P^{*}=\right.$ saturation partial pressure $=114$ ppt benzene under ambient conditions), the concentration dependencies of the responses of the two films were of similar form, with an approximately linear response observed at the lowest vapor concentrations (see Figure 3 inset). Above $P / P * \approx 0.81$, the response profile of the $50 \%(\mathrm{w} / \mathrm{w})$ carbon black-PEVA film remained continuous, but a significant increase in response was observed for the 15 
$\%(w / w)$ carbon black-PEVA film, consistent with swelling passing the latter material through its percolation threshold.

A further investigation into the effect of changing the conductor/insulator ratio of a sensor film was performed using a series of PVP and PEVA films with varying stoichiometries. Figure 4 shows the responses of the PVP films to $11 \mathrm{ppt}$ methanol and of the PEVA films to $9 \mathrm{ppt}$ benzene; baseline conductances for these sensors are also shown. The error bars in these graphs depict the variances in the data observed for a group of composite films at each specific carbon black content. As the carbon black contents of the films were lowered toward their respective percolation thresholds, the baseline conductances of the composites decreased. Furthermore, the magnitude of the maximum relative differential resistance response, $\Delta R_{\max , \infty} / R$, observed in response to introduction of a constant partial pressure of analyte increased as the conductor/insulator ratio decreased. The increase in response was significant, with $\Delta R_{\max , \infty} / R$ varying by a factor of five in response to changes in the carbon black content of the composite. Even larger improvements are expected with further reduction in the carbon black content, but to date, we have only studied films having initial baseline resistances less than the $10 \mathrm{M} \Omega$ limit of our multiplexing ohmmeter.

\section{Array-Based Vapor Sensing}

\section{a.Response Patterns for Various Vapors}

Although each individual sensor element had a characteristic relative differential resistance response, such data from an isolated sensor element would only be useful in a controlled environment that contained a single, known gas species. In more complex situations, data from a number of different sensors would be required. For this purpose, resistance data were obtained for arrays of carbon blackpolymer composite sensor elements during exposure to various chemically different gaseous species.

To evaluate the performance of a modestly sized sensor array, a set of 17 carbon blackpolymer composites was fabricated, with each sensor element having a different polymer in the composite (see Table 1a). Modified capacitors served as substrates for the composite films in the sensor array. Air (at a flow rate of $1 \mathrm{~L} \mathrm{~min}^{-1}$ ) saturated with one of nine common organic solvents: acetone, benzene, chloroform, ethanol, ethyl acetate, hexane, 2-propanol, methanol, or toluene was combined with a background air flow (6 L $\min ^{-1}$ ) and the mixture then introduced into a chamber containing the sensor array. This produced the following concentrations of each solvent: acetone: $49 \pm 2 \mathrm{ppt}$; benzene: $17.1 \pm 0.7$ ppt; chloroform: $40 \pm 2 \mathrm{ppt}$; ethanol: $10.0 \pm 0.4$ ppt; ethyl acetate $16.6 \pm 0.6 \mathrm{ppt}$; hexane: $29 \pm 1$ ppt; methanol: 23.0 $\pm 0.8 \mathrm{ppt}$; 2-propanol: $7.4 \pm 0.3 \mathrm{ppt}$; toluene: $4.7 \pm 0.2 \mathrm{ppt}$. Solvent vapors were introduced for $60 \mathrm{~s}$, in random order, six times each (except for acetone, for which only three exposures were performed), over a total period of $10 \mathrm{~h}$. Between vapor exposures, the sensors were exposed only to the solvent-free background flow (air) for a minimum of $6 \mathrm{~min}$, although shorter recovery times could have been employed in most cases. The data obtained from this experiment are summarized in Table 2.

Figure 5 displays the normalized, relative differential resistance data for this array during exposure to three representative solvents: methanol, ethyl acetate and benzene. These three solvents have similar vapor pressures $(108,81$, and 83 torr at $295 \mathrm{~K}$, respectively) but the solvents clearly differ in their chemical properties. To facilitate comparison between various sensors, a normalized signal, $S_{s j}^{\prime}$ 


$$
S_{s j}^{\prime}=\frac{\Delta R_{s j, \text { max }} / R_{j}}{\sum_{s}\left(\Delta R_{s j, \text { max }} / R_{j}\right)},
$$

has been plotted, where $s=$ methanol, ethyl acetate, or benzene, $j$ is the sensor number, $R_{j}$ is the baseline resistance of sensor $j$ before exposure to the solvent, and $\Delta R_{s j, \max }$ is the largest differential resistance change observed for the $j$ th sensor during the $60 \mathrm{sec}$ exposure to solvent $s$. For the film thicknesses and solvent concentrations used in this series of experiments, almost none of the sensors reached equilibrium so the recorded $\Delta R_{s j \text {, max }}$ value also incorporated temporal aspects of the resistance response of the array. Nevertheless, the histogram in Figure 5 clearly shows that the differential resistance response patterns generated by these solvents at this test pressure can be easily distinguished from each other using this modestly-sized sensor array.

The error bars in Figure 5 represent the standard error over the various exposures to each solvent (Table 2). These experiments were conducted at much higher vapor concentrations than those in Figure 2. At these higher concentrations, small decreases in responses and/or shifts in baselines were observed upon repeated solvent exposures of certain composites, but the small baseline shifts could be compensated for electronically if so desired and such minor shifts did not preclude the use of the sensor array, even under these stressing conditions, to separate the various vapors based on their array responses. The error bars depicted in Figure 5 reflect this effect, and also incorporate errors due to instabilities in our flow system and random errors in the resistance measurements. The presence of impurities in the background air stream, such as oil vapor from the compressed air source used to provide the carrier gas flow, would only minimally affect the data of Figure 5 since any signals arising from the presence of such impurities would be present in the resistance readings taken before and after exposure to the test vapors. Additionally, a slow baseline drift was also noted for most sensors. Over a three month period under ambient conditions, the baseline resistances of the composites in our 17-element array increased an average of $16 \%$, with the maximum increase being 55\% (for poly(vinyl chloride - co - vinyl acetate)) and the minimum being $<1 \%$ (poly(methyl vinyl ether - co maleic anhydride), although this baseline drift did not significantly affect the $\Delta R / R$ performance of the sensor array.

\section{b.Principal Component Analysis for Data Reduction of an Array Response}

A more quantitative approach to evaluating the performance of the sensor array is provided by principal component analysis. Principal component analysis transforms multivariate data sets into a coordinate space that allows for the variance in the data to be represented in the minimum number of dimensions. The vectors in this new coordinate set are the principal components of the data stream, and the separation between various vapors (e.g., various presentations to the array) is therefore readily visualized in this transformed data space. $^{31,32}$

The principal components are linear combinations of descriptors (in our case, the relative differential resistance responses):

$$
\mathbf{P}=\mathbf{D C},
$$

where $\mathbf{D}=\left\{d_{i j}\right\}$ and $\mathbf{P}=\left\{p_{i j}\right\}$ are $m \times n$ matrices and $\mathbf{C}=\left\{c_{i j}\right\}$ is an $n \times n$ matrix containing the coefficients of the linear combination. For a sensor array (with $n$ sensors) exposed $m$ times to various analytes, $d_{i j}$ represents the response of the $j$ th sensor to the $i$ th exposure and $p_{i j}$ the $j$ th principal component for the $i$ th exposure. The power of principal component analysis stems from the fact that the coefficient matrix, $C$, (containing 
as its columns the eigenvectors of the covariance matrix $\mathbf{D}^{\mathrm{T}} \mathbf{D}$ ) is chosen such that the principal components are mutually orthogonal, even though the original descriptors may have been heavily correlated.

Prior to performing principal component analysis, the data from the 17-element sensor array were normalized and autoscaled. The maximum differential resistance change for the $j$ th sensor to the $i$ th exposure, $\Delta R_{i j, \max }$, was normalized by the sum of the responses for all 17 sensors to that same exposure to produce a value $S_{i j}$ :

$$
S_{i j}=\frac{\Delta R_{i j, \max }}{\sum_{j} \Delta R_{i j, \max }}
$$

where $S_{i j}$ is the normalized signal. This normalization involves a summation over the entire array for a given exposure rather than over a collection of exposures for a given sensor, as in eq 1 . The normalization of eq 3 helps correct for differences in the exposure concentrations, which are a consequence of the solvents' differing vapor pressures. In the limit of linear response, the normalization process assures that the solvents are not being distinguished on the basis of their concentrations alone. The normalized maximum relative differential resistance changes were then autoscaled, resulting in a set of descriptors, $d_{i j}$, that were defined as:

$$
d_{i j}=\frac{S_{i j}-\bar{S}_{j}}{\sigma_{j}}
$$

Here, $\bar{S}_{j}$ and $\sigma_{j}$ are the mean and standard deviation, respectively, of all of the normalized signal responses of sensor $j$ to the entire range of solvents. This autoscaling procedure provides a means of accounting for differences in the dynamic ranges of the sensors. After normalizing and autoscaling, the data were transformed into principal component space. The principal components were numbered in accord with the amount of variance they contained: the lower the number, the more variance contained along that direction in principal component space.

The first five principal components (Figure 6) contained greater than $98 \%$ of the total variance in the data. The patterned areas in Figure 6 encompass all of the responses of the array that were produced during the repeated exposures to each specified vapor. The representation in principal component space clearly shows not only that, at the test concentrations used in this work, the carbon black-polymer composite array can readily distinguish nonpolar from polar solvents (e.g., benzene or toluene from methanol or acetone) but also illustrates that such an array can readily distinguish members of a related class of materials (e.g. methano1 from ethanol from 2-propanol, or benzene from toluene). A notable feature of this type of sensing device is that the sensor elements were not designed a priori to have specific responses to any particular vapor or class of vapors, yet the array could nevertheless separate a broad range of chemical species having relatively subtle differences in their chemical/physical properties.

\section{c.Array Response to Mixtures}

The ability of our sensor array to analyze vapor mixtures was also of interest. To explore this property, the sensor array was exposed to varying vapor concentrations of ethanol and methanol, and then to mixtures of these two vapors. To accomplish this, varying flow rates of air saturated with methanol and/or air saturated with ethanol were mixed into a $10 \mathrm{~L}$ $\min ^{-1}$ vapor-free air flow. For the mixtures, both the total flow rate of the methanol/ethanol analyte stream and the relative amounts of methanol to ethanol in the stream were varied. In the analysis of these data, the maximum relative differential resistance changes from the 
sensor array, $\Delta R_{\max } / R$, were not normalized according to eq 3 since the concentration dependence was also of significance in these experiments. Instead, the data (presented in Table 3 ) were simply autoscaled according to eq 4 and then transformed into principal component space. In these experiments, the variation in the analyte flow rates of the various mixtures at a given total analyte concentration was so small relative to the background flow rate that autoscaling of the data was still valid.

The first two principal components of this data set (containing $94 \%$ of the total variance in the data) are shown in Figure 7. The sensor array could distinguish methanol from ethanol at any of the concentrations studied and was also able to quantify the concentration of these vapors. In addition, for the mixtures, the data defined two distinct (pseudo-linear) paths that spanned the region between the responses of the pure components. Each path contained the data for a given total analyte flow rate, and the position along either path indicated the methanol/ethanol ratio of the mixture. Hence, the sensor array was also able to quantify the absolute concentration of each species in this binary mixture over the tested concentration range.

\section{Classification and Identification of Vapors Using the Carbon Black- Organic Polymer Chemiresistor Array}

\section{a. General Features of the Chemiresistor Array}

The success of modestly-sized arrays of chemically sensitive resistors in the detection and classification of vapors underscores the advantages of an approach to chemical sensing that utilizes broadly responsive sensing elements. The use of a common conducting phase, combined with the use of conventional insulating organic polymers to achieve the differential swelling properties of the various sensor elements, allows fabrication of such arrays from readily available, stable materials. An additional attractive feature of the present system is the simplicity of the signal transduction process. A chemical sorption event is directly transduced into an electrical resistance signal that can be readily integrated with inexpensive, conventional, signal processing circuitry.

Despite the lack of chemical specificity in the binding of an analyte to an individual array element, the carbon black-polymer composite chemiresistor array discriminated between a variety of vapors, some of which displayed very subtle chemical differences. This array also was able to identify and quantify the vapor mixture tested in this initial study. In fact, the 17-element carbon black-based sensor array was able to distinguish all of the nine test analytes from each other at the specific test concentrations used in this work, even though this test set required distinguishing molecules from very different classes, such as alcohols from aromatics, as well as those within a particular class, such as benzene from toluene or methanol from ethanol from 2-propanol.

The ability to resolve various vapors is quantified by their separation in principal component space. The best resolved vapors generally showed the largest separation in the early principal components, i.e., in those components containing the most variance in the data. For exposure of our array to the nine test analytes at the test concentrations used in this work, the first five principal components (shown in Figure 6) contained $49 \%, 25 \%$, $17 \%, 4 \%, 2 \%$, and $1 \%$, respectively, of the total variance. The positions of the various data points in the principal component space depicted in Figure 6 therefore need to be scaled by the relative magnitudes of each principal component in order to obtain a true visualization of the resolving power of the sensor array. The greatest resolution was observed between the polar compounds, which were distinguished in the first three dimensions 
of principal component space (Figure 6a). This is reasonable because nearly all of the sensor elements were reasonably polar, with many being able to participate in hydrogen bonding, so gas-solid interactions based on polarity dominated the binding of the various analytes into the composite films of the sensor array. The more non-polar molecules were separated collectively from the polar compounds in the first three principal components, but separation of the non-polar compounds from each other was based on more subtle effects. These effects only become evident through an analysis of the higher principal components of the sensor array response (see Figure 6b). Of course, principal component analysis is a purely statistical approach to data reduction, and a neural network could easily be trained, without additional array design, to assign an increased weighting to the response of certain sensors if the primary function of the array were, for example, to separate benzene from toluene. Even restricting the data evaluation to principal component analysis, resolution of non-polar analytes should improve significantly with the incorporation of additional sensor elements having composite films fabricated from carbon black and non-polar organic polymers.

Ideally, the swelling-induced relative differential resistance response of each of the chemiresistors could be related to solubility parameters that correlate with the partition coefficients for binding of a given vapor into a given polymer film. Such a correlation has been drawn for the swelling of a commercial carbon black-polymer composite with a variety of saturated vapors. ${ }^{14}$ For some of our chemiresistors, the relative differential resistance response did indeed track with the extent of swelling predicted by solubility parameters. For other chemiresistors, however, the agreement between maximum relative differential resistance changes and solubility parameters was poor. There was some difficulty in drawing definitive conclusions from our experiments because the vapor concentrations for the nine solvents were different (thus requiring correction based on linear response) and because the short exposure times used to investigate the array responses to various vapors did not permit equilibrium to be reached on the sensor elements at the test concentrations and composite film thicknesses used in this study. However, as long as the exposure period was maintained constant, the data of Figures 5 and 6 show that the various vapors could be distinguished even without reaching an equilibrium differential resistance signal (which could be obtained in a specified time period through use of thinner films, if so desired). The data of Figure 5 do qualitatively show the selectivity of the sensors for different solvents and demonstrate that these responses agree with simple chemical ideas. For instance, the $\Delta R_{\max } / R$ response of the protic poly (4-vinyl phenol) composite, sensor 1, to methanol was 55 times greater than that its response to benzene. The situation is reversed for one of the non-polar sensor elements, PEVA (sensor 16), with benzene producing a 42 times larger $\Delta R_{\max } / R$ signal than methanol. Note that although it is possible to analyze the data of Table 2 to ascertain which subset of sensor elements provided the "best" discrimination for a given pair of vapors, this assessment is very task-dependent (i.e., the "best" subset of sensors for separating benzene from toluene are different from the "best" subset of sensors for separating methanol from ethanol and are different yet again from the "best" subset of sensors for separating benzene and toluene in the presence of methanol or ethanol, etc.), so this type of evaluation has not been performed extensively at this time.

Further improvements in the resolving power of the sensor array are expected when the temporal information provided by each solvent is incorporated into the data analysis algorithm. The time course of the resistance change is a 
potentially valuable additional discriminant because it will reflect the diffusion rate of a vapor into a particular film. In fact, the concentration of $\mathrm{CHCl}_{3}$ or $\mathrm{CCl}_{4}$ above a poly(vinyl chloride)-carbon black composite has been determined previously on the basis of temporal response information alone and potentially can take advantage of data involving specific molecular interactions that affect the binding and diffusion kinetics of various analytes into the sensor elements ${ }^{16}$ We are currently investigating the best means by which test analytes can be classified by our sensor array; however, since it is unlikely that a single algorithm will be optimal for all tasks on a given sensor array, we have not pursued this scenario-specific analysis extensively at the present time.

\section{b.Identification of Mixtures and Distinguishing Unknowns from Mixtures of Previously Identified Vapors}

The identification and quantification of methanol/ethanol mixtures by the sensor array highlights further the potential power of arraybased sensing, provided that linearity is maintained or that extensive calibration runs are performed over nonlinear response regions. Determining both the ratio of components in, and the total concentration of, a binary mixture necessarily requires more than a single degree of freedom. Although such additional degrees of freedom could be achieved with a single sensor, for instance by using temporal information, they are much more easily incorporated into a multicomponent architecture such as in the array structure described herein. The data of Figure 7 also show that, for methanol/ethanol mixtures, the separation between methanol and ethanol vapors in principal component space is maintained for several different concentrations of these vapors both separately and in binary mixtures. Of course, evaluation of the quantitative changes in separation factors in principal component space that might occur for all possible analytes of interest at all possible concentration ranges of practical interest is beyond the scope of this initial investigation. Clearly, the separation ability of such arrays contemplated for any specific practical application must be evaluated for the task of concern under application-specific conditions.

A particularly interesting question that naturally arises in the context of environmental monitoring is whether a sensor array can distinguish a chemically distinct species from a mixture of vapors for which the array response has been previously investigated. A multilinear regression was thus performed to determine if the array response of a given member of our collection of test solvents could be expressed as a linear combination of the responses of the other test solvents. In this evaluation, the best fit parameters, $\boldsymbol{a}=\left\{a_{1}\right.$, $\left.a_{2}, \ldots, a_{8}\right\}$, to the following system of linear equations were determined using a constrained multi-linear regression: ${ }^{33}$

$$
a_{1} \mathbf{r}_{1}+a_{2} \mathbf{r}_{2}+\ldots+a_{8} \mathbf{r}_{8}=\mathbf{r}_{9}
$$

The column vector, $\mathbf{r}_{i}$, contained the maximum relative differential resistance responses of the seventeen sensors to a particular solvent, and the index 9 indicated the solvent to be expressed as a linear combination of the other eight vapors. In the limit of linear response, the coefficients, $\boldsymbol{a}$, are proportional to the partial pressures of the solvents. Consequently, there is a limited range of physically relevant values of $\boldsymbol{a}$. For example, coefficients that represent mixtures containing negative concentrations of any vapor are nonphysical and can be rejected. Similarly, if one knew the total vapor concentration, or had some independently determined, physically realistic constraints on the mass balance or on the concentrations of any of the components in the mixture, additional ranges of $a$ could be identified as invalid solutions to the problem of concern and thus also rejected. 
In determining the best fit to eq 5 with our sensor array, the coefficients $a$ were merely constrained to remain positive, since negative coefficients for our data set implied negative pressures. No other constraints, either on the total pressure of the system or on the pressure limits of a particular species, were applied. Using only this simple constraint criterion, seven of the test solvents at the test concentrations used in this work could be conclusively identified as unique species that were not mixtures of the other solvents. For example, Figure 8 demonstrates the best fit for ethyl acetate in terms of the responses of the array to the other vapors in the test set. The best fit pattern to the ethyl acetate response was generated by a mixture of acetone, benzene and chloroform $(840: 82: 1)$. Although this mixture could account for the response of several sensor elements, the complete pattern could not be satisfactorily matched over the entire array. Similar behavior was observed for acetone, chloroform, ethanol, hexane, 2propanol, and methanol in our system.

Benzene and toluene were the only two solvents that produced responses at the test concentration under study which could be modeled as a linear combination of the maximum relative differential resistance response patterns generated by the other solvents in our test set. For example, the fingerprint of benzene could be successfully modeled as a linear combination of the response produced by toluene combined with responses arising from small concentrations of the other solvents. Use of further information in the data produced by the chemiresistor array, such as the temporal response of the resistance signals, might resolve even these remaining ambiguities. The ability to distinguish chemically distinct species from mixtures of other vapors is a much stronger indication of the information content of the sensor array responses than simply separating individual analytes of similar concentrations, since the inclusion of mixtures and varying analyte concentrations as possible allowed solutions introduces many more degrees of freedom in fitting the data produced by exposure to the unknown vapor. The ability to distinguish chemically distinct test vapors from any physically realistic mixtures of other predetermined vapors further demonstrates the potential of carbon black-organic polymer sensor arrays for environmental monitoring, where the identification of foreign matter is often crucial.

We also note that for many applications that require evaluation of the constancy of complex vapor mixtures, such as for example quality control applications of foodstuffs, linearity of the sensor array response to analyte concentration is not particularly important, whereas constancy of signal response and signal/noise limits are more critical. In contrast, other vapor detection applications might demand different performance specifications. Although it is clear that these polymer composite chemiresistor materials make promising candidates for sensor arrays, it is not clear at this point which applications are best matched to the performance factors that can be achieved from these types of systems.

\section{Response Mechanisms of Individual Carbon Black-Polymer Composite Sensor Elements}

a. Correlations Between Resistance Changes
and Predictions of Percolation Theory

The resistivity vs. carbon black content of carbon black-organic polymer composites is well described by percolation theory. ${ }^{7,9,34-36}$ At low carbon black loadings, the composites are insulators because no connected pathway of conductive particles exists across the material. As the carbon black content is increased, a sharp transition occurs in which the resistivity of the composite can decrease dramatically (by up to 10 orders of magnitude) with a small variation in the carbon black 
concentration. At this transition point, designated as the percolation threshold, a connected pathway of carbon black particles is formed. A consistent explanation of the differential resistance response of our sensor elements to solvent vapor is that swelling disrupts the conduction pathways, thereby resulting in an increased resistance of the composite film.

More quantitatively, percolation theory predicts that the resistivity of a carbon black-organic polymer composite, $\rho$, will be given by: ${ }^{14,34}$

$$
\begin{gathered}
\rho=\frac{(z-2) \rho_{c} \rho_{m}}{A+B+\left[(A+B)^{2}+2(z-2) \rho_{c} \rho_{m}\right]^{1 / 2}}, \\
\text { where } \\
\quad \begin{array}{c}
A=\rho_{c}\left[-1+(z / 2)\left(1-\left(v_{c} / f\right)\right)\right], \\
B=\rho_{m}\left[\left(z v_{c} / 2 f\right)-1\right],
\end{array}
\end{gathered}
$$

and where $\rho_{c}$ is the resistivity of the carbon black, $\rho_{m}$ is the resistivity of the polymer matrix, $v_{c}$ is the volume fraction of carbon black in the composite, $z$ is the coordination number of the carbon black particles, and $f$ is their total packing fraction $\left(v_{c} \leq f\right)$. The volume fraction of carbon black in the composite at the percolation threshold, $v_{p}$, is given by $2 f / z$. Figure 9 displays the theoretical prediction of eq 6 for a hypothetical composite having $v_{p}=0.33$ and $\rho_{\mathrm{m}} / \rho_{\mathrm{c}}=$ $10^{11}$, under the assumption that swelling does not affect the volume of the conductive element but only changes the total volume, $V$. In this situation, swelling of the insulating phase will produce a relative volume change, $\Delta V / V$, of the film.

In Figure 9, two primary response regions can be identified. For volume changes small enough that the carbon black volume fraction in the swollen composite, $v_{c}{ }^{s w}$, remains greater than $v_{p}$, swelling reduces, but does not eliminate, connected conductance pathways in the film. The relative differential resistance response in this regime is pseudo-linear over a reasonable volume range of the composite (c.f. Figure 9 inset). However, for swellinginduced volume changes such that $v_{c}>v_{p}>v_{c} s w$, the resistivity of the swollen film is predicted to be much larger than that of the denser, unswollen composite material.

Experimentally, profiles such as those in Figure 9 can be related to the maximum relative differential resistance response data under at least two separate experimental protocols: (1) measuring the time dependence of the resistance change upon swelling by a given solvent vapor, or (2) determining the resistance changes after reaching equilibrium in response to exposure to a series of different concentrations of a given vapor. For swelling of individual carbon black-organic polymer composites by organic liquids or vapors, approach (1) has been demonstrated previously to generate time-dependent resistance changes that are in qualitative agreement with the predictions of Figure 9. ${ }^{15}$ This approach only can be applied when the resistivity of the carbon-black composite is spatially uniform during the swelling process; i.e., when the diffusion rate of vapor through the film is much greater than the rate of swelling of the composite. In the work described herein, approach (2) has been adopted since it allows a comparison of theory with the maximum relative differential resistance response observed under equilibrium conditions, $\Delta R_{\max , o d} R$ (Figure 3). The similar functional form of the predicted response (Figure 9) and the experimental data (Figure 3) argues strongly that the swelling-induced relative differential resistance responses of the carbon black-polymer composite chemiresistors are dominated by a percolation mechanism. Note that in our experiments, only those composites having the lowest carbon black loadings, and exposed to the highest vapor concentrations, (i.e. the 15\% PEVA chemiresistor exposed to air that was nearly saturated with benzene) swelled sufficiently to ensure that $v_{c}>v_{p}>v_{c} s w$. This behavior is also in agreement with the 
theoretical predictions of Figure 9, in which the swelling required to reach the critical increase in resistance is predicted to be the lowest for the composite with the lowest initial conducting phase content.

The majority of the studies reported herein were restricted to composites in swelling environments where $v_{c} s w$ remained greater than $v_{p}$. For conductive composites swelling in the range $v_{c}{ }^{s w}>v_{p}$, percolation theory predicts that the maximum relative differential resistance signal produced at equilibrium in response to a given level of swelling will increase with decreasing carbon black content. This behavior is illustrated in Figure 10 using eq 6 with $\rho_{\mathrm{m}} / \rho_{\mathrm{c}}=10^{11}, v_{p}=0.33$ and assuming a constant $1 \%$ swelling for the various measurements. Comparison of Figure 4 with Figure 10 demonstrates that the PEVA and PVP composites investigated herein behaved in qualitative agreement with the predictions of percolation theory for films in which $v_{c} s w>v_{p}$. Thus, the magnitude of the $\Delta R_{\max , \infty} / R$ response to a given swelling change can be manipulated through carbon black content even for composites operating under conditions in which $v_{c}{ }^{S w}>v_{p}$.

\section{b. Sensitivity Estimates for Vapor Detection Using Carbō̄ Black-Polymer Composite Chemiresistor Arrays}

From the data in Figures 3 and 4, it is possible to estimate the ultimate sensitivities possible with the sensing approaches discussed above. The largest maximum relative differential resistance signal observed in response to a change in partial pressure, $\Delta P$, of a test vapor is expected for a composite having its stoichiometry poised such that the slightest swelling will pass the material through the percolation threshold. Although none of the composites synthesized in this work met this criterion, the sharp increase in response observed for the 15\% PEVA composite above
$P / P^{*}=0.81$ can be used to estimate the partial pressure dependence of the maximum relative differential resistance response expected for such a situation (Figure 3 ). Increasing the benzene pressure from $P / P^{*}=0.81$ to 0.84 corresponded to the introduction of an additional $3.4 \mathrm{ppt}$ of benzene. The composite resistance increased by a factor of five in response to this change in vapor pressure, implying that $\left(\Delta R_{\max , \infty} / R\right) / \Delta P$ would be greater than $100 \%$ per ppt, i.e. $>1 \mathrm{ppt}^{-1}$ (assuming a linear resistance vs. swelling response over this range of swelling) This is much larger than the $\left(\Delta R_{\max , \infty} / R\right) / \Delta P$ response observed for swelling of composites having $v_{c}{ }^{s w}>v_{p}$. For example, the $15 \%$ PEVA sensor's response to $10 \mathrm{ppt}$ of benzene was $10 \%$, yielding a $\left(\Delta R_{\max , \infty} R\right) / \Delta P$ response of $0.01 \mathrm{ppt}^{-1}$. Data for the other sensor/solvent combinations studied in this work (for a $60 \mathrm{~s}$ exposure period) can be obtained by scaling these sensitivity values by the relative responses displayed by each sensor/solvent system (Table 2).

Of course, the useable information arising from a sensor element is not a function of the signal amplitude alone but depends instead on the signal/noise ratio. Shurmer et al. have discussed the ultimate sensitivity attainable with resistance-based vapor sensors in the limit of Johnson or white noise. ${ }^{37}$ For application in arrays, the lower limit on the measurable voltage was placed at ten times the noise voltage. For a $\left(\Delta R_{\max , \infty} / R\right) / \Delta P$ value of 0.25 $\mathrm{ppt}^{-1}$, typical of $\mathrm{SnO}_{2}$ vapor sensors, the calculated lower detection limit was $1 \mathrm{ppb}$ (ppb $=$ part per billion $(\mathrm{v} / \mathrm{v}))$ of solvent vapor. Empirically, however, somewhat higher sensitivity limits of $10-100 \mathrm{ppb}$ were estimated from experimental data using $\mathrm{SnO}_{2}$ vapor sensors at a signal/noise level of 10:1. Using the same approach as Shurmer et al., the 1 $\mathrm{ppt}^{-1}\left(\Delta R_{\max , \infty} / R\right) / \Delta P$ response of our carbon black composites predicts a lower vapor detection limit of $0.25 \mathrm{ppb}$ at a signal/noise of 
$10: 1$ in the most favorable case where the sensor and amplifier noise is purely limited by Johnson noise. For comparison, composites in which $v_{c} s w>v_{p}$, i.e., those for which swelling does not induce the composite to cross its percolation threshold, the observed responses of $\approx 0.01-\mathrm{ppt}^{-1}$ imply Johnsonnoise-limited vapor detection levels of $\approx 25$ $\mathrm{ppb}$ at a signal/noise ratio of 10:1.

The above limits can, of course, only be taken as crude estimates that might be obtained under optimized conditions. The actual signal/noise limits will depend on the acceptable power levels that can be used in the measurement, thermal and temporal drifts, the validity of linear response (especially given the extrapolations made above), and on the other sources of noise such as interference or $1 / \mathrm{f}$ noise, which is characteristic of carbon blackcomposite resistors. ${ }^{38}$ We have performed some sensitivity studies with our current equipment, and observe that the achievable measurement resolution, rather than noise, limits our sensitivity at present. For example, for a circuit with a $40 \mathrm{k} \Omega$ base resistance, our $\mathrm{dc}$ resistance measurement resolution is currently $0.025 \%$. For a $55 \%$ carbon black PVP chemiresistor having such a baseline resistance and having its noise less than our measurement resolution, we have been able to sense $70 \mathrm{ppm}(\mathrm{ppm}=$ part per million $(\mathrm{v} / \mathrm{v}))$ levels of methanol (through a $0.05 \%$ maximum relative differential resistance change). This measurement was recorded without the possible sensitivity benefits afforded by working very near the percolation threshold. We are currently fully characterizing the noise in our chemiresistors and improving our measurement techniques to thoroughly investigate the sensitivity limits attainable with carbon black composite films.

An advantage of using sensing elements whose conductivity is dominated by percolation is that their sensing properties can be readily controlled through changes in the composition of the composite. By working near the percolation threshold, such that $v_{c} s w$ drops below $v_{p}$ at the slightest swelling, very good sensitivity could, in principle, be achieved in response to small changes in vapor pressure (with a loss of linearity in the resistance vs. vapor concentration profile, however for a large range of vapor concentrations). Another approach would be to use composites with stoichiometries such that $v_{c} s w$ passed through $v_{p}$ after some amount of swelling. The focus here would not be on measuring the actual resistance of the composite as $v_{c} s w$ dropped below $v_{p}$; rather, it would be on determining if $v_{c}{ }^{s w}$ becomes less than $v_{p}$ upon swelling. In other words, each composite would provide a binary piece of data that, when coupled with many composites of differing stoichiometries, would determine the degree of swelling. Of course, the resolution of such a system depends on the number of stoichiometries included. Consequently, the size of an array including many different types of polymers might become prohibitively large unless the film deposition process can be automated and miniaturized. The final approach suggested by the form of $\Delta R / R$ vs. $\Delta V / V$ shown in Figure 9 , and the one used in our sensor array, is to use composites such that $v_{c}{ }^{s w}$ remains greater than $v_{p}$ upon swelling. Although this may not afford the sensitivity of exclusively using composites with $v_{c}$ slightly above $v_{p}$, the lower resistivities of the composites utilized herein permit the utilization of thin films to obtain rapid response times. This allows operation in the linear response range in order to utilize the principle of superposition to analyze unknown patterns produced by the array, and allow use of a relatively inexpensive multiplexing digital multimeter to monitor the data arising from the array elements.

\section{B.Poly(pyrrole) Composite Sensors}

\section{Properties of the Poly(pyrrole) Films}


A key to our ability to fabricate chemically diverse sensing elements is the preparation of processable, air stable films of electrically conducting organic polymers. This was achieved through the controlled chemical oxidation of pyrrole (PY) using phosphomolybdic acid $\left(\mathrm{H}_{3} \mathrm{PMo}_{12} \mathrm{O}_{40}\right)(20)$ in tetrahydrofuran:

$$
\begin{aligned}
& \mathrm{PY} \rightarrow \mathrm{PY}^{+}+\mathrm{e}^{-} \\
& 2 \mathrm{PY}^{+} \rightarrow \mathrm{PY}_{2}+2 \mathrm{H}^{+} \\
& \mathrm{H}_{3} \mathrm{PMo}_{12} \mathrm{O}_{40}+2 \mathrm{e}^{-}+2 \mathrm{H}^{+} \rightarrow \mathrm{H}_{5} \mathrm{PMo}_{12} \mathrm{O}_{40}
\end{aligned}
$$

The redox-driven or electrochemically-induced polymerization of pyrrole has been explored previously, but this process typically yields insoluble, intractable deposits of poly(pyrrole) as the product. $^{39}$ Our approach was to use low concentrations of the $\mathrm{H}_{3} \mathrm{PMo}_{12} \mathrm{O}_{40}$ oxidant $\left(\mathrm{E}^{\mathrm{o}}=+0.36 \mathrm{~V}\right.$ vs. SCE $) .{ }^{40}$ Since the electrochemical potential of $\mathrm{PY}^{+} / \mathrm{PY}$ is more positive $\left(\mathrm{E}^{\circ}=+1.30 \mathrm{~V} \text { vs. } \mathrm{SCE}\right)^{41}$ than that of $\mathrm{H}_{3} \mathrm{PMo}_{12} \mathrm{O}_{40} / \mathrm{H}_{5} \mathrm{PMo}_{12} \mathrm{O}_{40}$, the equilibrium concentration of $\mathrm{PY}^{+}$, and thus the rate of polymerization, was relatively low in dilute solutions (0.19 M PY, 0.09 $\mathrm{M} \mathrm{H}_{3} \mathrm{PMo}_{12} \mathrm{O}_{40}$ ). However, it has been shown that the oxidation potential of pyrrole oligomers decreases from $+1.20 \mathrm{~V}$ to +0.55 to $+0.26 \mathrm{~V}$ vs. SCE as the number of units increase from one to two to three, and that the oxidation potential of bulk poly(pyrrole) occurs at $-0.10 \mathrm{~V}$ vs. SCE. ${ }^{42}$ As a result, oxidation of pyrrole trimers by phosphomolybdic acid is expected to be thermodynamically favorable. This allowed processing of the monomer-oxidant solution (i.e. spin coating, dip coating, introduction of insulating polymers, etc.), after which time polymerization to form thin films was simply effected by evaporation of the solvent. The dc electrical conductivity of poly(pyrrole) films formed by this method on glass slides, after rinsing the films with methanol to remove excess phosphomolybdic acid and/or monomer, was on the order of $15-30 \mathrm{~S}-\mathrm{cm}^{-1}$ for films ranging from $40-100 \mathrm{~nm}$ in thickness.

The poly(pyrrole) films produced in this work exhibited excellent electrochemical and optical properties. For example, the cyclic voltammetric behavior of a chemically polymerized poly(pyrrole) film showed a cathodic wave at $-0.40 \mathrm{~V}$ which corresponded to the reduction of poly(pyrrole) to its neutral, nonconducting state, and an anodic wave at $-0.20 \mathrm{~V}$ which corresponded to the reoxidation of poly(pyrrole) to its conducting state. ${ }^{43}$ The lack of additional faradaic current, which would result from the oxidation and reduction of phosphomolybdic acid in the film, suggested that the Keggin structure of phosphomolybdic acid was not present in the film anions ${ }^{44}$ and implies that $\mathrm{MoO}_{4}{ }^{2-}$, or other anions, served as the poly(pyrrole) counterions in the polymerized films. The optical spectra of these films were also in accord with expectations for poly(pyrrole), with the processed film displaying an absorption band at $4.0 \mathrm{eV} .45,46$

As described in the experimental section, various insulating polymers were introduced into the polymer films (Table $1 b$ ). This allowed chemical control over the binding properties and electrical conductivity of the resulting polymer composites. Sensor arrays consisted of as many as 14 different elements, with each element synthesized to produce a distinct chemical composition, and thus a distinct sensor response, for its polymer film. The resistance, $R$, of each film-coated individual sensor was automatically recorded before, during, and after exposure to various odorants. A typical trial consisted of a $60 \mathrm{sec}$ rest period in which the sensors were exposed to flowing air (3.0 liter- $\left.\mathrm{min}^{-1}\right)$, a $60 \mathrm{sec}$ exposure to a mixture of air (3.0 liter- $\mathrm{min}^{-1}$ ) and air that had been saturated with solvent $\left(0.5-3.5\right.$ liter- $\left.\min ^{-1}\right)$, and then a $240 \mathrm{sec}$ exposure to air (3.0 liter- $\left.\min ^{-1}\right)$. 


\section{Array-Based Vapor Sensing}

In an initial processing of the data, the only information used was the maximum amplitude of the resistance change divided by the initial resistance, $\Delta R_{\text {max }} / R_{i}$, of each individual sensor element. Most of the sensors exhibited either increases or decreases in resistance upon exposure to different vapors, as expected from changes in the polymer properties upon exposure to different types of chemicals. ${ }^{47,48}$ However, in some cases, sensors displayed an initial decrease followed by an increase in resistance in response to a test odor. Since the resistance of each sensor could increase and/or decrease relative to its initial value, two values of $\Delta R_{\max } / R_{i}$ were reported for each sensor. The source of the bi-directional behavior of some sensor/odor pairs has not yet been studied in detail, but in most cases this behavior arose from the presence of water (which by itself induced rapid decreases in the film resistance) in the reagent-grade solvents used to generate the test odors of this study. The observed behavior in response to these airexposed, water-containing test solvents was reproducible and reversible on a given sensor array, and the environment was representative of many practical odor sensing applications in which air and water would not be readily excluded.

Figure 11b-d depicts representative examples of sensor amplitude responses of a sensor array (Table 1b). In this experiment, data were recorded for 3 separate exposures to vapors of acetone, benzene, and ethanol flowing in air. It is readily apparent that these odorants each produced a distinctive response on the sensor array. In additional experiments, a total of 8 separate vapors (acetone, benzene, chloroform, ethanol, isopropyl alcohol, methanol, tetrahydrofuran, and ethyl acetate), chosen to span a range of chemical and physical characteristics, were evaluated over a
5 day period on a 14-element sensor array (Table $1 \mathrm{~b}$ ). As discussed below, each odorant could be clearly and reproducibly identified from the others using this sensor apparatus.

Principal component analysis ${ }^{31}$ was used to simplify presentation of the data and to quantify the distinguishing abilities of individual sensors and of the array as a whole. In this approach, linear combinations of the $\Delta R_{\text {max }} / R_{i}$ data for the elements in the array were constructed such that the maximum variance [defined as the square of the standard deviation] was contained in the fewest mutually orthogonal dimensions. This allowed representation of most of the information contained in data sets shown in Figure 11b-d in two (or three) dimensions. The resulting clustering, or lack thereof, of like exposure data in the new dimensional space was used as a measure of the distinguishing ability, and of the reproducibility, of the sensor array.

In order to illustrate the variation in sensor response of individual sensors that resulted from changes in the insulating polymer, principal component analysis was performed on the individual, isolated responses of each of the 14 individual sensor elements in a typical array (Figure 12). Since each individual sensor produced two data values, principal component analysis of these responses resulted in only two orthogonal principal components, $\mathrm{pc} 1$ and $\mathrm{pc} 2$. As an example of the selectivity exhibited by an individual sensor element, the sensor designated as number 5 in Figure 12 (which was mixed with poly(styrene)) confused acetone with chloroform, isopropyl alcohol, and tetrahydrofuran. It also confused benzene with ethyl acetate, while easily distinguishing ethanol and methanol from all other solvents. Changing the insulating polymer to poly( $\alpha$-methyl styrene) (sensor number 6 in Figure 12) had little effect on the spatial distribution of the responses with respect to one another and with respect to the 
origin. Thus, as expected, a rather slight chemical modification of the insulating polymer had little effect on the relative variance of the eight test odorants. In contrast, the addition of a cyano group to the insulating polymer, in the form of poly(styreneacrylonitrile), (sensor number 7 in Figure 12), resulted in a larger contribution to the overall variance by benzene and chloroform, while decreasing the contribution of ethanol. Changing the substituent group in the insulating polymer to a hydrogen bonding acid (poly(styrene-allyl alcohol), sensor number 9 in Figure 12) increased the contribution of acetone to the overall variance while having little effect on the others odors, with the exception of confusing methanol and ethanol. These results suggest that the behavior of the sensors can be systematically altered by varying the chemical composition of the insulating polymer.

Figure 13 shows the principal component analysis for some of the 14 sensors described in Table $1 \mathrm{~b}$ and Figures 11 and 12. When the solvents were projected into a three dimensional odor space (Figure 13a or 13b), all eight solvents were easily distinguished with the specific array discussed herein. Detection of an individual test odor, based only on the criterion of observing $\sim 1 \% \Delta \mathrm{R}_{\max } / \mathrm{R}_{\mathrm{i}}$ values for all elements in the array, was readily accomplished with no control over the temperature or humidity of the flowing air. Further increases in sensitivity are likely after a thorough utilization of the temporal components of the $\Delta R_{\text {max }} / R_{i}$ data as well as a more complete characterization of the noise in the array.

We have also investigated the suitability of this sensor array for identifying the components of certain test mixtures. This task is greatly simplified if the array exhibits a predictable signal response as the concentration of a given odorant is varied, and if the responses of various individual odors are additive (i.e. if superposition is maintained). When a 19element sensor array was exposed to a number, $n$, of different acetone concentrations in air, the $\left(\mathrm{CH}_{3}\right)_{2} \mathrm{CO}$ concentration was semiquantitatively predicted from the first principal component. This was evident from a good linear least square fit of the partial pressure of acetone, $\mathrm{P}_{\mathrm{a}}$ (torr), in air with the first principal component. The acetone concentration could be more accurately predicted using a multilinear least square fit through the first three principal components.

The same sensor array was also able to resolve the components in various test methanolethanol mixtures. A linear relationship was observed between the first principal component and the mole fraction of methanol in the liquid phase, $x_{\mathrm{m}}$, in a $\mathrm{CH}_{3} \mathrm{OH}-\mathrm{C}_{2} \mathrm{H}_{5} \mathrm{OH}$ mixture, demonstrating that superposition held for this mixture/sensor array combination. Furthermore, although the components in the mixture could be predicted fairly accurately from just the first principal component, an increase in the accuracy could be achieved using a multi-linear least square fit through the first three principal components. This relationship held for $\mathrm{CH}_{3} \mathrm{OH} /\left(\mathrm{CH}_{3} \mathrm{OH}+\right.$ $\mathrm{C}_{2} \mathrm{H}_{5} \mathrm{OH}$ ) ratios of 0 to 1.0 in air-saturated solutions of this vapor mixture. The conducting polymer-based sensor arrays could therefore not only distinguish between pure test vapors, but also allowed analysis of concentrations of odorants as well as analysis of binary mixtures of certain test vapors.

In summary, the results presented herein provide a basis for advances in the area of odor sensor design. A relatively simple array design, using only a multiplexed low-power $\mathrm{dc}$ electrical resistance readout signal, has been shown to readily distinguish between various test odorants. Such conducting polymer-based arrays are simple to construct and modify, and afford an opportunity to effect chemical control over the response pattern of a vapor. For 
example, by increasing the ratio of insulating polymer to conducting polymer, it should be possible to approach the percolation threshold, at which point the conductivity exhibits a very sensitive response to the presence of the sorbed molecules. Furthermore, producing thinner films will afford the opportunity to obtain decreased response times, and increasing the number of insulating polymers and polymer backbone motifs will likely result in increased diversity among sensors. This type of chemical control, with its accompanying simplicity of signal transduction and readout, is the main novel feature of these odor sensors. Such systems also hold potential for evaluating the generality of neural network algorithms that are currently being developed to understand how the mammalian olfactory system identifies the directionality, concentration, and identity of various odors.

\section{CONCLUSIONS}

Broadly responsive, easily monitored vapor sensor have been developed using thin film, conductor-polymer composites. The chemiresistor elements, making use of carbon black or poly(pyrrole) as the conducting phase, have been shown to give distinctive, lowpower, dc, signal patterns in response to the presence of test concentrations of various organic solvent vapors. The response mechanism of the sensors made with carbon black as the conductor has been shown to agree qualitatively with predictions of percolation theory. An understanding of the response mechanism allows for the sensor response signals to be deliberately tuned to a desirable range by changing the nature and abundance of the insulating polymer and/or the abundance of carbon black in the sensor film. Also described is a simple chemical polymerization of poly(pyrrole), allowing it to be cast in thin films. These types of sensors are inexpensive and easily fabricated. Furthermore, the ease with which they can be modified as well as customized for specific chemical and environmental monitoring tasks makes them potentially attractive for such applications.

\section{ACKNOWLEDGMENTS}

This work was supported in part by the Caltech Consortium in Chemistry and Chemical Engineering, the E.I. DuPont de Nemours and Company, Inc. and the Eastman Kodak Company, and by the National Aeronautics and Space Administration and the National Science Foundation, grant CHE9202583. M.C.L. acknowledges Caltech for an Arthur Amos Noyes Fellowship and B.J.D. acknowledges the Natural Science and Engineering Research Council of Canada for a 1967 Centennial Fellowship and the O'Brien Foundation for financial support. We thank Profs. J.J. Hopfield and J.M. Bower, and the members of their research groups, for helpful discussions.

\section{REFERENCES}

(1)Reed, R.R. Neuron 1992, 8, 205.

(2)Lancet, D.; Ben-Arie, N. Current Biology 1993, 3, 668 .

(3)Zaromb, S.; Stetter, J.R. Sensors and Actuators 1984, 6, 225.

(4)Lundstrom, I.; Erlandsson, R.; Frykman, U.; Hedborg, E.; Spetz, A.; Sundgren, H.; Welin, S.; Winquist, F. Nature 1991, 352, 47.

(5)Shurmer, H.V.; Gardner, J.W. Sensors And Actuators B Chemical 1992, 8, 1 .

(6)Gardner, J.W.; Bartlett, P.N. Sensors And Actuators B Chemical 1994, 18, 211.

(7)Norman, R.H. Conductive Rubbers and Plastics; Elsevier: Amsterdam, 1970.

(8)Carbon Black-Polymer Composites; ; Sichel, E.K., Ed. Eds.; Marcel Dekker, Inc.: New York, 1982.

(9)Medalia, A.I. Rubber Chemistry and Technology 1986, 59, 432.

(10)Ford, C.J. In U.S. Patent 2,691,134, 1951 
(11)Newton, R.G. Journal of Rubber Research 1946, 15, 35.

(12)Sands, A.G.; McDowell, M.V. Rubber Age, New York 1956, 80, 500.

(13)Boyd, J.; Bulgin, D. J. Text. Inst. Proc. $1957,48,66$.

(14)Lundberg, B.; Sundqvist, B. Journal of Applied Physics 1986, 60, 1074.

(15)Ruschau, G.R.; Newnham, R.E.; Runt, J.; Smith, B.E. Sensors And Actuators 1989, $20,269$.

(16)Talik, P.; Zabkowskawaclawek, M.; Waclawek, W. Journal Of Materials Science 1992, 27, 6807.

(17)Ballantine, D.S.; Rose, S.L.; Grate, J.W.; Wohltjen, H. Analytical Chemistry 1986, 58, 3058.

(18)Grate, J.W.; Abraham, M.H. Sensors and Actuators $B$ Chemical 1991, 3, 85.

(19)Grate, J.W.; Rosepehrsson, S.L.; Venezky, D.L.; Klusty, M.; Wohltjen, H. Analytical Chemistry 1993, 65, 1868.

(20)Nakamoto, T.; Fukuda, A.; Moriizumi, T. Sensors and Actuators B Chemical 1993, 10,85 .

(21)Gardner, J.W.; Shurmer, H.V.; Corcoran, P. Sensors And Actuators $B$ Chemical 1991, 4, 117.

(22)Gardner, J.W.; Shurmer, H.V.; Tan, T.T. Sensors And Actuators B Chemical 1992, 6, 71 .

(23)Corcoran, P.; Shurmer, H.V.; Gardner, J.W. Sensors And Actuators B Chemical $1993,15,32$.

(24)Shurmer, H.V.; Corcoran, P.; Gardner, J.W. Sensors And Actuators B Chemical 1991, 4, 29.

(25)Pearce, T.C.; Gardner, J.W.; Friel, S.; Bartlett, P.N.; Blair, N. Analyst 1993, 118, 371.

(26)Freund, M.S.; Lewis, N.S. Proceedings of the National Academy of Sciences, USA 1995, 92, 2652.

(27)Merrifield, R.B. Angewandte Chemie $1985,24,799$.
(28)Nunez, E.M.; Myerson, A.S.; Kwei, T.K. Polymer Engineering and Science 1991, $31,1172$.

(29)Sarin, V.K.; Kent, S.B.; Mitchell, A.R.; Merrifield, R.B. Journal of the American Chemical Society 1984, 106, 7845.

(30)Merrifield, R.B. Makromol. Chem. $1988,19,31$.

(31)Hecht, H.G. Mathematics in Chemistry: An Introduction to Modern Methods; Prentice Hall: Englewood Cliffs, NJ, 1990.

(32)Gardner, J.W. Sensors And Actuators B Chemical 1991, 4, 109.

(33)Spath, H. Mathematical Algorithms For Linear Regression; Academic Press: Boston, 1991.

(34)Kirkpatrick, S. Reviews of Modern Physics 1973, 45, 574.

(35)Jachym, B.J. In Carbon Black - Polymer Composites; Sichel, E.K. Ed..; Marcel Dekker, Inc.: New York, 1982; p 103.

(36)Reboul, J.P. In Carbon Black-Polymer Composites; Sichel, E.K. Ed..; Marcel Dekker, Inc.: New York, 1982; p 79.

(37)Shurmer, H.V.; Corcoran, P.; James, M.K. Sensors And Actuators B Chemical 1993, 16, 256.

(38)Brophy, J.J. Basic Electronics for Scientists; McGraw-Hill: New York, 1972.

(39)Salmon, M.; Kanazawa, K.; Diaz, A.F.; Krounbi, M. Journal of Polymer Science Part C Polymer Letters 1982, 20, 187.

(40)Pope, M.T. Heteropoly and Isopoly Oxometalates; Springer: New York, 1983.

(41)Andrieux, C.P.; Auddebert, P.; Hapiot, P.; Saveant, J.-M. Journal of the American Chemical Society 1990, 112, 2439.

(42)Diaz, A.F.; Crowley, J.; Bargon, J.; Gardini, G.P.; Torrance, J.B. Journal of Electroanalytical Chemistry 1981, 121, 355.

(43)Kanazawa, K.K.; Diaz, A.F.; Krounbi, M.T.; Street, G.B. Synthetic Metals 1981, 4, 119.

(44)Bidan, G.; Genies, E.M.; Lapkowski, M. Journal of Electroanalytical Chemistry 1988, $251,297$. 
(45)Yakushi, K.; Lauchan, L.J.; Clarke, T.C.; Street, G.B. Journal of Chemical Physics 1983, 79, 4774.

(46)Kaufman, J.H.; Colaneri, N.; Scott, J.C.; Street, G.B. Physical Review Letters 1984, 53, 1005.
(47)Topart, P.; Josowicz, M. Journal of Physical Chemistry 1992, 96, 7824. (48)Charlesworth, J.M.; Partridge, A.C.; Garrard, N. Journal of Physical Chemistry 1993, 97, 5418. 
1 poly(4-vinyl phenol)

2 poly(styrene - co - allyl alcohol), 5.7\% hydroxyl

$3 \operatorname{poly}(\alpha$-methylstyrene)

4 poly(vinyl chloride - co - vinyl acetate), $10 \%$ vinyl acetate

5 poly(vinyl acetate)

$6 \operatorname{poly}(N$-vinylpyrrolidone $)$

7 poly(carbonate bisphenol A)

$8 \quad$ poly(styrene)

9 poly(styrene - co - maleic anhydride), $50 \%$ styrene

10 poly(sulfone)

11 poly(methyl methacrylate)

12 poly(methyl vinyl ether - co - maleic anhydride)

13 poly(vinyl butyral)

14 poly(vinylidene chloride - co - acrylonitrile), $80 \%$ vinylidene chloride

15 poly(caprolactone)

16 poly(ethylene - co-vinyl acetate), $82 \%$ ethylene

$17 \quad$ poly(ethylene oxide) 
Table 1b- Polymers used in poly(pyrrole) array*

\begin{tabular}{cl} 
sensor & plasticizer \\
\hline 1 & none \\
2 & none** \\
3 & poly(styrene) \\
4 & poly(styrene) \\
5 & poly(styrene) \\
6 & poly(a-methyl styrene) \\
7 & poly(styrene-acrylonitrile) \\
8 & poly(styrene-maleic anhydride) \\
9 & poly(styrene-allyl alcohol) \\
10 & poly(vinyl pyrrolidone) \\
11 & poly(vinyl phenol) \\
12 & poly(vinyl butyral) \\
13 & poly(vinyl acetate) \\
14 & poly(carbonate) \\
\hline
\end{tabular}

* Sensors contained 2:3 (w:w) ratio of pyrrole to insulating polymer.

** Film not rinsed to remove excess phosphomolybdic acid. 
Table 2. Relative differential resistance changes $(\Delta R / R)$, in percent, for each sensor element exposed to nine solvents. The numbers of the sensor elements correspond to the polymer composites indicated in Table 1a. Values are averages of six exposures to each solvent, except for acetone, for which only three exposures were performed. Errors are in parentheses.

\begin{tabular}{|c|c|c|c|c|c|c|}
\hline Element -> & 1 & 2 & 3 & 4 & 5 & 6 \\
\hline toluene & $0.13(9)$ & $0.3(1)$ & $1.2(2)$ & $0.4(2)$ & $0.05(2)$ & $0.63(6)$ \\
\hline methanol & $10 .(1)$ & $1.6(1)$ & $1.9(1)$ & $0.37(3)$ & $1.05(3)$ & $16 .(1)$ \\
\hline 2-propanol & $0.23(9)$ & $0.17(2)$ & $0.52(9)$ & $0.08(3)$ & $0.02(2)$ & $1.6(5)$ \\
\hline hexane & $0.06(3)$ & $0.13(3)$ & $0.8(2)$ & $0.28(7)$ & $0.01(2)$ & $0.18(6)$ \\
\hline ethyl acet. & $1.2(1)$ & $0.66(7)$ & $2.2(2)$ & $0.76(6)$ & $0.14(3)$ & $2.1(4)$ \\
\hline ethanol & $2.3(7)$ & $0.4(1)$ & $1.3(3)$ & $0.11(5)$ & $0.09(2)$ & $5 .(2)$ \\
\hline chloroform & $0.45(7)$ & $0.70(8)$ & $2.2(3)$ & $0.9(2)$ & $0.35(8)$ & $5 .(1)$ \\
\hline benzene & $0.20(6)$ & $0.35(8)$ & $1.4(4)$ & $0.50(9)$ & $0.09(3)$ & $1.0(2)$ \\
\hline acetone & $4.2(2)$ & $1.0(2)$ & $3.3(1)$ & $0.67(8)$ & $0.19(2)$ & $2.8(1)$ \\
\hline Element $->$ & 7 & 8 & 9 & 10 & 11 & 12 \\
\hline toluene & $1.9(4)$ & $2.3(2)$ & $0.07(4)$ & $1.4(2)$ & $1.3(5)$ & $0.08(4)$ \\
\hline methanol & $1.2(1)$ & $0.82(5)$ & $2.3(2)$ & $3.5(5)$ & $2.0(2)$ & $2.3(4)$ \\
\hline 2-propanol & $0.6(1)$ & $0.52(6)$ & $0.06(2)$ & $0.6(1)$ & $0.7(2)$ & $0.08(3)$ \\
\hline hexane & $0.9(2)$ & $1.4(2)$ & $0.03(2)$ & $0.7(2)$ & $0.6(2)$ & $0.01(2)$ \\
\hline ethyl acet. & $3.6(7)$ & $4.9(6)$ & $0.39(3)$ & $4.0(5)$ & $3.4(1)$ & $0.31(8)$ \\
\hline ethanol & $0.9(2)$ & $0.7(2)$ & $0.4(1)$ & $1.6(6)$ & $1.3(3)$ & $0.4(1)$ \\
\hline chloroform & $3.7(2)$ & $6.9(7)$ & $0.26(5)$ & $3.4(4)$ & $6 .(1)$ & $0.15(6)$ \\
\hline benzene & $2.2(5)$ & $3.0(3)$ & $0.10(3)$ & $1.9(2)$ & $1.7(5)$ & $0.07(3)$ \\
\hline acetone & $5.6(9)$ & $5.6(6)$ & $0.77(4)$ & $5.0(1)$ & $3.5(1)$ & $0.51(4)$ \\
\hline Element -> & 13 & 14 & 15 & 16 & 17 & \\
\hline toluene & $0.07(3)$ & $0.9(2)$ & $4.2(2)$ & $19 .(1)$ & $1.47(6)$ & \\
\hline methanol & $0.75(4)$ & $1.3(1)$ & $1.2(1)$ & $0.9(2)$ & $0.74(6)$ & \\
\hline 2-propanol & $0.02(2)$ & $0.21(6)$ & $0.9(2)$ & $1.5(4)$ & $0.3(1)$ & \\
\hline hexane & $0.05(3)$ & $0.22(5)$ & $1.1(1)$ & $16.0(9)$ & $0.70(4)$ & \\
\hline ethyl acet. & $0.07(4)$ & $2.2(2)$ & $3.3(2)$ & $7.3(6)$ & $0.88(9)$ & \\
\hline ethanol & $0.02(4)$ & $0.7(2)$ & $0.9(3)$ & $1.1(4)$ & $0.5(1)$ & \\
\hline chloroform & $0.08(6)$ & $1.3(3)$ & $15 .(2)$ & $56 .(6)$ & $5 .(1)$ & \\
\hline benzene & $0.06(4)$ & $1.01(8)$ & $5.3(3)$ & $21 .(1)$ & $1.5(1)$ & \\
\hline acetone & $0.08(5)$ & $2.89(3)$ & $2.94(3)$ & $4.3(6)$ & $0.80(8)$ & \\
\hline
\end{tabular}


Table 3. Relative differential resistance changes $(\Delta R / R)$, in percent, for each sensor element exposed to methanol/ethanol mixtures. The numbers of the sensor elements correspond to the polymer composites indicated in Table 1a; sensor element number 1 was defective at this point and is therefore not included in this tabulation. The concentrations of the components of the mixtures are given in parts per thousand (ppt).

\begin{tabular}{ccccccccc} 
Exp. & ppt MeOH & ppt EtOH & element 2 & element 3 & element 4 & element 5 & element 6 & element 7 \\
\hline 1 & $9.2(3)$ & 0 & 0.892 & 1.142 & 0.332 & 0.679 & 4.194 & 0.653 \\
2 & $7.8(3)$ & 0 & 0.803 & 1.023 & 0.332 & 0.594 & 3.695 & 0.579 \\
3 & $6.5(2)$ & $0.53(3)$ & 0.804 & 0.957 & 0.193 & 0.566 & 3.493 & 0.550 \\
4 & $5.2(2)$ & $1.06(6)$ & 0.625 & 0.877 & 0.249 & 0.396 & 2.957 & 0.490 \\
5 & $3.9(1)$ & $1.65(9)$ & 0.535 & 0.824 & 0.166 & 0.424 & 2.719 & 0.490 \\
6 & $2.58(9)$ & $2.3(1)$ & 0.491 & 0.878 & 0.235 & 0.283 & 2.546 & 0.505 \\
7 & $1.25(5)$ & $2.8(1)$ & 0.402 & 0.744 & 0.138 & 0.170 & 2.071 & 0.446 \\
8 & 0 & $3.3(2)$ & 0.223 & 0.638 & 0.152 & 0.028 & 1.656 & 0.416 \\
9 & 0 & $3.9(2)$ & 0.268 & 0.718 & 0.222 & 0.057 & 1.833 & 0.431 \\
10 & 0 & $2.7(1)$ & 0.223 & 0.585 & 0.125 & 0.057 & 1.478 & 0.342 \\
11 & $6.3(2)$ & 0 & 0.714 & 0.904 & 0.332 & 0.509 & 3.197 & 0.505 \\
12 & 0 & $2.0(1)$ & 0.223 & 0.452 & 0.083 & 0.028 & 1.246 & 0.297 \\
13 & $4.0(1)$ & $0.34(2)$ & 0.268 & 0.479 & 0.083 & 0.141 & 1.363 & 0.297 \\
14 & $3.2(1)$ & $0.67(4)$ & 0.313 & 0.559 & 0.000 & 0.170 & 1.540 & 0.282 \\
15 & $2.4(9)$ & $1.01(5)$ & 0.357 & 0.612 & 0.208 & 0.198 & 1.719 & 0.312 \\
16 & $1.59(6)$ & $1.35(7)$ & 0.447 & 0.692 & 0.166 & 0.283 & 1.956 & 0.372 \\
17 & $0.79(3)$ & $1.69(9)$ & 0.536 & 0.745 & 0.180 & 0.396 & 2.313 & 0.401 \\
18 & $4.7(2)$ & 0 & 0.581 & 0.745 & 0.166 & 0.396 & 2.430 & 0.431 \\
19 & $3.2(1)$ & 0 & 0.447 & 0.612 & 0.236 & 0.283 & 1.896 & 0.312 \\
20 & $1.62(6)$ & 0 & 0.313 & 0.426 & 0.055 & 0.198 & 1.186 & 0.238 \\
21 & 0 & $1.36(7)$ & 0.179 & 0.399 & 0.097 & 0.028 & 0.890 & 0.223 \\
22 & 0 & $0.69(4)$ & 0.089 & 0.240 & 0.055 & 0.000 & 0.534 & 0.119
\end{tabular}

Exp. ppt $\mathrm{MeOH}$

1

2

3

4

$9.2(3)$

$7.8(3)$

$6.5(2)$

$5.2(2)$
$3.9(1)$

$2.58(9)$

$1.25(5)$

0

0

0

6.3(2)

0

4.0(1)

$3.2(1)$

$2.4(9)$

$1.59(6)$

$0.79(3)$

$4.7(2)$

$3.2(1)$

$1.62(6)$

0

0

ppt EtOH

element 8

element 9

element 9 element 10 ele

$0 \quad 0.490$

1.401

0.429

$0.53(3)$

0.417

$1.06(6)$

0.417

0.368

0.393

0.343

0.319

0.319

0.270

0.368

1.278

0.909

0.815

1.091

0.796

0.841

0.717

0.682

0.592

0.644

0.374

0.607

0.218

0.569

0.493

$3.9(2)$

0.249

0.531

0.249

0.417

1.154

0.721

0.125

0.341

0.221

$0.34(2)$

0.270

$0.67(4)$

0.270

$1.35(7)$

0.295

$1.69(9)$

0.344

0.344

0.270

0.172

0.246

0.312

0.379

0.437

0.379

0.530

0.418

0.624

0.494

0.749

0.570

0.905

0.569

0.686

0.418

0.374

0.266

0.031

0.304

0.062

0.190

$0.69(4)$

0.123

lement 11

0.764

0.669
0.628

0.519

0.491

0.437

0.382

0.328

0.355

0.300

0.573

0.219

0.246

0.273

0.328

0.410

0.437

0.465

0.328

0.191

0.191

0.109

$\frac{\text { element }}{0.403}$

0.293

0.183

0.110

0.146

0.073

0.110

0.037

0.110

0.037

0.183

0.110

0.037

0.110

0.147

0.073

0.183

0.183

0.110

0.110

0.074

0.037

element 13

0.312

0.349

0.342

0.134

0.193

0.089

0.149

0.030

0.104

0.045

0.386

0.015

0.164

0.074

0.104

0.104

0.268

0.238

0.164

0.178

0.045

0.045 


\begin{tabular}{ccccccc} 
Exp. & ppt $\mathrm{MeOH}$ & ppt EtOH & element 14 & element 15 & element 16 element 17 \\
\hline 1 & $9.2(3)$ & 0 & 0.738 & 0.873 & 0.386 & 0.617 \\
2 & $7.8(3)$ & 0 & 0.708 & 0.798 & 0.361 & 0.561 \\
3 & $6.5(2)$ & $0.53(3)$ & 0.615 & 0.778 & 0.337 & 0.532 \\
4 & $5.2(2)$ & $1.06(6)$ & 0.574 & 0.664 & 0.313 & 0.436 \\
5 & $3.9(1)$ & $1.65(9)$ & 0.534 & 0.664 & 0.361 & 0.437 \\
6 & $2.58(9)$ & $2.3(1)$ & 0.472 & 0.627 & 0.385 & 0.428 \\
7 & $1.25(5)$ & $2.8(1)$ & 0.370 & 0.627 & 0.385 & 0.386 \\
8 & 0 & $3.3(2)$ & 0.205 & 0.590 & 0.433 & 0.376 \\
9 & 0 & $3.9(2)$ & 0.205 & 0.705 & 0.481 & 0.408 \\
10 & 0 & $2.7(1)$ & 0.185 & 0.534 & 0.313 & 0.301 \\
11 & $6.3(2)$ & 0 & 0.678 & 0.724 & 0.337 & 0.506 \\
12 & 0 & $2.0(1)$ & 0.185 & 0.420 & 0.265 & 0.270 \\
13 & $4.0(1)$ & $0.34(2)$ & 0.247 & 0.458 & 0.265 & 0.291 \\
14 & $3.2(1)$ & $0.67(4)$ & 0.308 & 0.477 & 0.265 & 0.279 \\
15 & $2.4(9)$ & $1.01(5)$ & 0.411 & 0.438 & 0.240 & 0.311 \\
16 & $1.59(6)$ & $1.35(7)$ & 0.493 & 0.496 & 0.240 & 0.332 \\
17 & $0.79(3)$ & $1.69(9)$ & 0.431 & 0.553 & 0.240 & 0.375 \\
18 & $4.7(2)$ & 0 & 0.514 & 0.553 & 0.264 & 0.374 \\
19 & $3.2(1)$ & 0 & 0.452 & 0.476 & 0.192 & 0.289 \\
20 & $1.62(6)$ & 0 & 0.247 & 0.267 & 0.144 & 0.193 \\
21 & 0 & $1.36(7)$ & 0.206 & 0.324 & 0.216 & 0.192 \\
22 & 0 & $0.69(4)$ & 0.062 & 0.190 & 0.120 & 0.128
\end{tabular}


Figure Captions:

Figure 1. Schematic of a chemiresistor sensor array and the response profiles generated by such an array. In this work, an array of 17 conducting carbon black-polymer composites has been used (the polymers of the composites are listed in Table 1a). The resistance of each composite is monitored and observed to increase upon swelling by organic vapors. The open arrow in the schematic is a time marker corresponding to the introduction of solvent vapor and the solid arrow to its removal. The maximum relative differential resistance changes $\left(\Delta R_{\max } / R\right)$ for the elements of the array during exposure to the test vapor provide a fingerprint that can be used to classify various analytes.

Figure 2. The resistances, $R$, of carbon black composites of (a) PEVA and (b) PVP upon 15 repeated exposures to benzene (at 1.1 ppt) and methanol (at $1.5 \mathrm{ppt}$ ), respectively. The PEVA composite was fabricated from a $15 \%(w / w)$ carbon black mixture and the PVP composite from a $45 \%$ (w/w) carbon black mixture. Both composite films were deposited onto glass slides. The exposure periods were for $15 \mathrm{~s}$ during which time the resistances increased as shown. These exposures were interlaced between recovery periods in which the resistances decreased. These traces demonstrate the good reproducibility and stability that can be achieved with carbon black composites.

Figure 3. The maximum relative differential resistance changes, $\Delta R_{\max , \infty} / R$, for two carbon black composites of PEVA (thin films on glass substrates) in response to varying partial pressures, $P$, of benzene. The exposure times for different concentrations of benzene varied, but in each case they were sufficient for the chemiresistor to realize its maximum resistance change in response to the test vapor. $P^{*}$ is the vapor pressure of benzene under ambient conditions, and the legend indicates the carbon black content of the fabrication solution. The film with the lower carbon black content passed through its percolation threshold upon swelling, resulting in a sharp increase in the relative differential resistance change at $P / P^{*} \approx 0.81$. The film with the greater carbon black content did not exhibit such critical behavior, in accord with expectations that the composite with the higher volume fraction of the conductive component should require more swelling to cross through its percolation threshold. The inset shows the data at low partial pressures on a linear scale to demonstrate the approximately linear dependence of $\Delta R_{\max , \infty} / R$ on $\Delta P$ in this regime.

Figure 4. The maximum relative differential resistance changes $\left(\Delta R_{\max , o d} R\right.$, solid circles, left axis) and baseline conductances ( $G$, open circles, right axis) for (a) PEVA- and (b) PVPcarbon black composites (thin films on glass substrates) as a function of carbon black content. For the PEVA sensors, the maximum relative differential resistance changes are those observed in response to $9 \mathrm{ppt}$ benzene; for the PVP sensors, they are those observed in response to $11 \mathrm{ppt}$ methanol. The error bars on the conductance values are estimates based on the deviation between four or five composite films fabricated at each composition, and the error bars of the $\Delta R_{\max , o d} R$ values are based on the deviation between the responses of these four or five sensors to four exposures each. At carbon black concentrations below those shown, the baseline resistances of the composites were too high to be measured by the multiplexing ohmmeter (>10 M $\Omega$ ) used in monitoring the array-based sensor.

Figure 5. The normalized signal response, $S^{\prime}$, of the 17 chemiresistors in the array (see Table 1a) for $60 \mathrm{~s}$ exposures to methanol, ethyl acetate, and benzene. The concentrations of each solvent vapor were: benzene: $17.1 \pm 0.7$ ppt; ethyl acetate 16.6 $10.6 \mathrm{ppt}$; methanol: 
$23.0 \pm 0.8 \mathrm{ppt}$. Each recorded $S$ value is the average of six separate exposures (Table 2). As a visualization aid, each sensor's relative differential resistance response was individually normalized by dividing the $\Delta R_{\max } / R$ value observed for exposure to a particular vapor by the sum of that sensor's $\Delta R_{\text {max }} / R$ responses to methanol, ethyl acetate, and benzene. The normalization factors (in \%) are given parenthetically following the sensor numbers. For instance, for sensor 1 , $\Delta R_{\max } / R$ was 0.1 (i.e., $11 \%$ ) in response to methanol. The fingerprints for the three solvents are clearly different, demonstrating the ability of this array to distinguish these vapors.

Figure 6. The results from the exposure of the 17-element array to nine solvents as represented in (a) the first three dimensions of principal component space, and (b) the third, fourth and fifth dimensions of principal component space. These five principal components contain over $98 \%$ of the total variance in the data. The concentrations of each solvent vapor were: acetone: $49 \pm 2 \mathrm{ppt}$; benzene: $17.1 \pm 0.7 \mathrm{ppt}$; chloroform: $40 \pm 2 \mathrm{ppt}$; ethanol: $10.0 \pm 0.4 \mathrm{ppt}$; ethyl acetate $16.6 \pm 0.6$ ppt; hexane: $29 \pm 1 \mathrm{ppt}$; methanol: $23.0 \pm 0.8$ ppt; 2-propanol: $7.4 \pm 0.3 \mathrm{ppt}$; toluene: $4.7 \pm 0.2$ ppt. Each patterned region contains six points corresponding to six exposures of each solvent (Table 2), except for acetone which only contained data for three exposures. The unresolved region in $\mathrm{PC} 1-\mathrm{PC} 2-\mathrm{PC} 3$ space contains the responses to benzene, chloroform, hexane, and toluene. Each loci of points for each solvent occupies a unique region of principal component space, indicating that the array distinguished all nine test analytes.

Figure 7. The first two principal components resulting from the exposure of a 16-element array to methanol (circles), ethanol (squares), and mixtures of the two ( + and $\mathbf{X}$ ). To expose the sensor, an air flow saturated with methanol (at a flow rate $\mathrm{Q}_{\mathrm{MeOH}}$ ) and/or one saturated with ethanol (at a flow rate QEtOH) were mixed into a $10 \mathrm{~L} \mathrm{~min}^{-1}$ background flow passing over the sensor array. The analyte flow rates, $\mathrm{QEtOH}_{\mathrm{E}}$ or $\mathrm{QMeOH}_{\mathrm{M}}$, for the pure solvents were $0.10,0.20,0.30,0.40,0.50$, and $0.60 \mathrm{~L} \mathrm{~min}^{-1}$ with the more filled symbols indicating the direction of increasing flow. For the mixtures, both the composition of the mixture and its total concentration were varied and exposures of solvents to the array were for $60 \mathrm{~s}$ each. Exposures with QMeOH : QEtOH ratios of $16: 84,33: 67,50: 50,67: 33$, and $84: 16$ were performed at two different total analyte flow rates: $\mathrm{QMeOH}+\mathrm{QEtOH}=0.30 \mathrm{~L}$ $\mathrm{min}^{-1}$ or $0.50 \mathrm{~L} \mathrm{~min}-1$. The direction of increasing mole fraction of methanol in the vapor mixture, $\chi_{\mathrm{M}}$, is indicated. In the limit of linear response, the mixtures are expected to fall on one of the two dotted lines, depending on the total analyte flow rate.

Figure 8. A histogram comparing the maximum relative differential resistance responses of the 17 sensors during a $60 \mathrm{~s}$ exposure to ethyl acetate to those predicted for a hypothetical mixture of acetone, benzene, and chloroform $(840: 82: 1)$. This mixture represents the best fit from a multi-linear regression where the maximum relative differential resistance responses to ethyl acetate were modeled as a linear combination of the responses observed during a $60 \mathrm{~s}$ exposure to each of the other eight solvents (see eq 6 and associated text). Although the maximum relative differential resistance response of some of the sensors could be accounted for, the entire fingerprint could not be satisfactorily modeled. The error bars on the observed data correspond to the variance in responses of similarly prepared sensors.

Figure 9. The relative differential resistance change, $\Delta R / R$, predicted by percolation theory (see eq 5 and associated text) as a function of the relative volume change, $\Delta V / V$, of a carbon 
black-polymer composite upon swelling. The volume of carbon black is assumed to be unaffected by swelling and the polymer matrix is assumed to have a conductivity 11 orders of magnitude lower than that of carbon black. The three separate lines are for composites with differing initial volume percentages of carbon black, as indicated. The percolation threshold for the system is at $v_{c}=0.33$. The total volume change results in a change in the effective carbon black content, $v_{c}{ }^{s w}$. When, $v_{c}{ }^{s w}$ drops below the percolation threshold, a sharp increase in response is observed. Of course, the position of this sharp increase depends on the value of $v_{c}$. The inset shows the resistance change on a linear scale for swelling in the region where $v_{c} s w$ remains greater than its value at the percolation threshold. Comparison of this figure with Figure 3 shows the qualitative agreement between the observed response and the predictions of percolation theory.

Figure 10. The baseline conductivity, $\sigma_{i}$, and relative differential resistance response, $\Delta R / R$, to $1 \%$ swelling predicted by percolation theory (see eq 5 and associated text) as a function of the initial volume fraction of carbon black, $v_{c}$. As in Figure 9, $v_{p}=0.33$ and $\rho_{\mathrm{m}} /$ $\rho_{c}=10^{11}$. The baseline conductivities are normalized by the conductance of pure carbon black, $\sigma_{c}$. The $1 \%$ swelling in this range of compositions is such that the effective carbon black content always remains above the percolation threshold. Comparison of this figure with Figure 4 demonstrates the qualitative agreement between percolation theory and the observed $\Delta R_{\max } / R$ responses for the carbon black composites studied in this work.

Figure 11. (A) Schematic of a sensor array showing an enlargement of one of the modified ceramic capacitors used as sensing elements. The response patterns generated by the sensor array described in Table $1 \mathrm{~b}$ are displayed for:
(B) acetone; (C) benzene; and (D) ethanol. The sensor response was defined as the maximum percent increase and decrease of the resistance divided by the initial resistance (gray bar and black bar respectively) of each sensor upon exposure to sollvent vapor. In many cases sensors exhibited reproducible increases and decreases in resistance. An exposure consisted of: i) a $60 \mathrm{sec}$ rest period in which the sensors were exposed to flowing air (3.0 liter- $\left.\mathrm{min}^{-1}\right)$; ii) a $60 \mathrm{sec}$ exposure to a mixture of air (3.0 liter- $\left.\mathrm{min}^{-1}\right)$ and air that had been saturated with solvent $\left(0.5\right.$ liter- $\left.\mathrm{min}^{-1}\right)$; and iii) a $240 \mathrm{sec}$ exposure to air (3.0 liter- $\mathrm{min}^{-1}$ ).

Figure 12. Principal component analysis of autoscaled data from individual sensors containing different insulating polymers: (A) poly(styrene); (B) poly( $\alpha$-methyl styrene); (C) poly(styrene-acrylonitrile); (D) poly(styreneallyl alcohol). Data were obtained from multiple exposures to acetone (a), benzene (b), chloroform (c), ethanol (e), isopropyl alcohol (i), methanol $(\mathrm{m})$, tetrahydrofuran $(\mathrm{t})$, or ethyl acetate (@) over a period of 5 days with the test vapors exposed to the array in various sequences. The numbers of the figures refer to the sensor elements described in Table $1 b$. The units along the axes indicate the amplitude of the principal component that was used to describe the particular data set for an odor. The black regions indicate data clusters corresponding to a single solvent which could be distinguished from all others; gray regions highlight data of solvents whose signals overlapped with others around it. Exposure conditions were identical to those in Figure 11.

Figure 13. Principal component analysis of data obtained from all sensors (Table $1 b$ ). Conditions and symbols are identical to Figure 12. Figure 13a shows data represented in the first three principal components $\mathrm{pc} 1, \mathrm{pc} 2$ and $\mathrm{pc} 3$, while Figure $13 \mathrm{~b}$ shows the data when represented in $\mathrm{pc} 1, \mathrm{pc} 2$, and $\mathrm{pc} 4$. A higher degree of discrimination between some solvents could be obtained by considering the 
fourth principal component as illustrated by a larger separations between chloroform, tetrahydrofuran, and isopropyl alcohol in Figure 13b. 


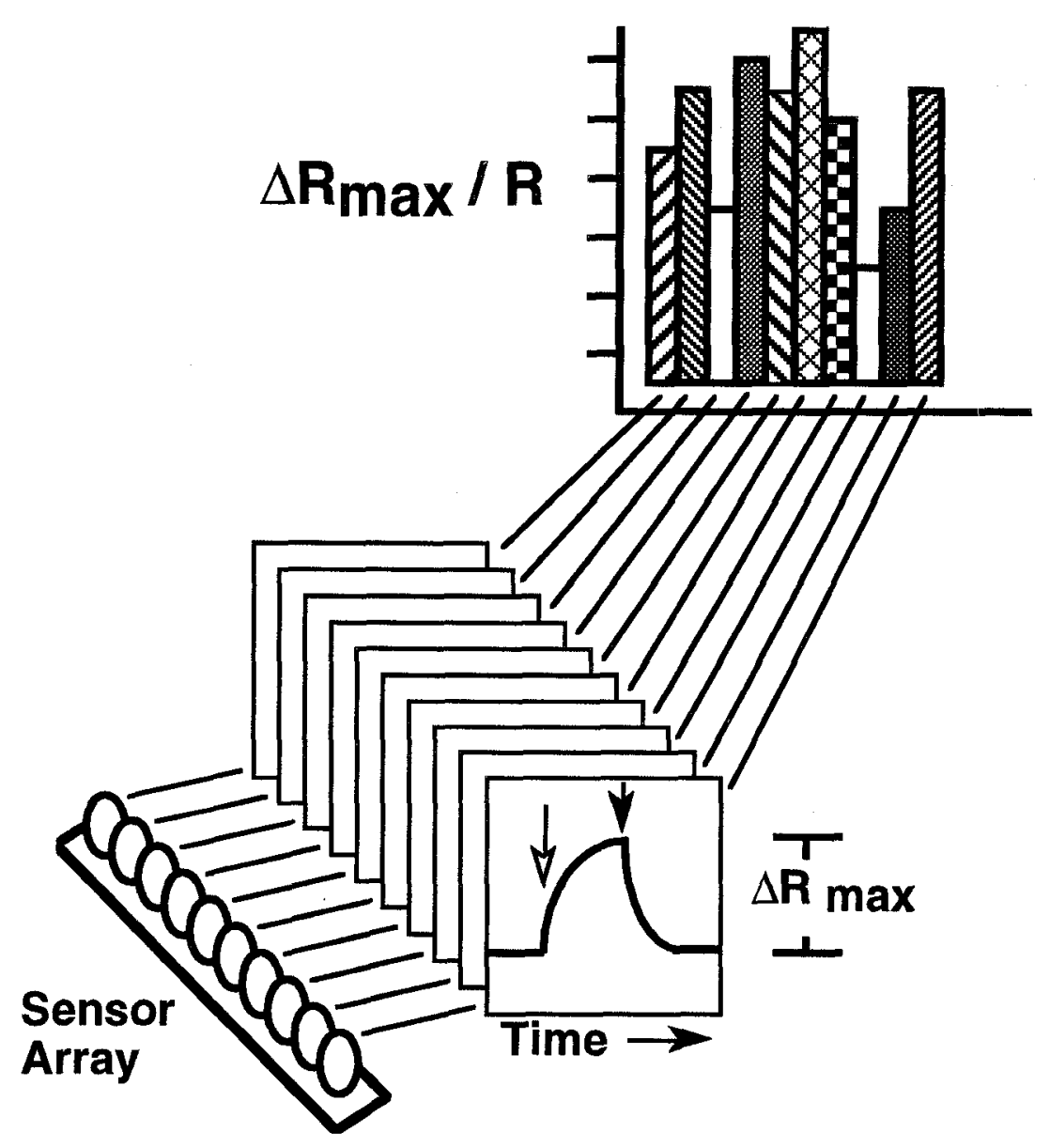

Lonergan et. al., Figure 1 


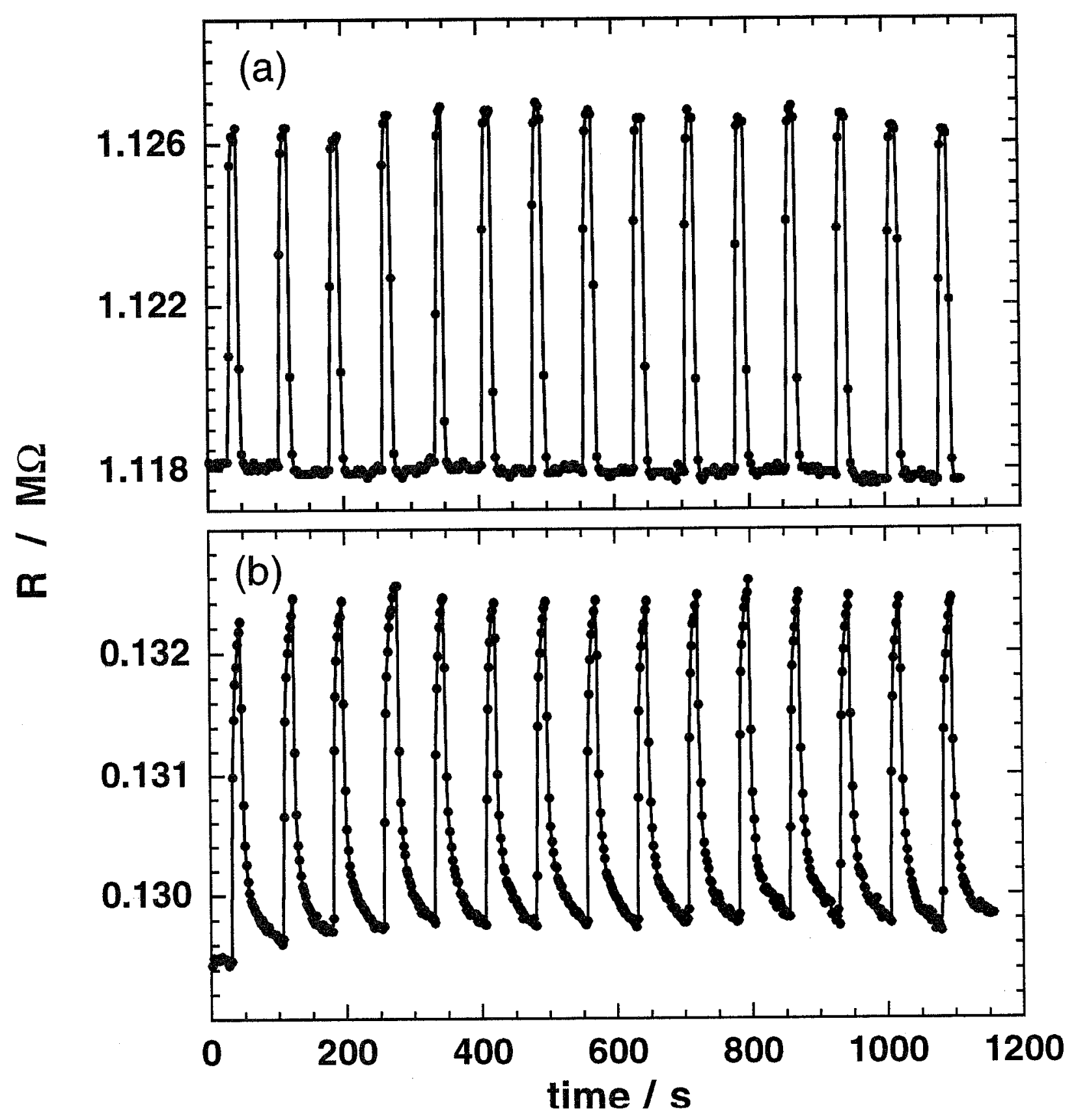

Lonergan et. al., Figure 2 a,k 


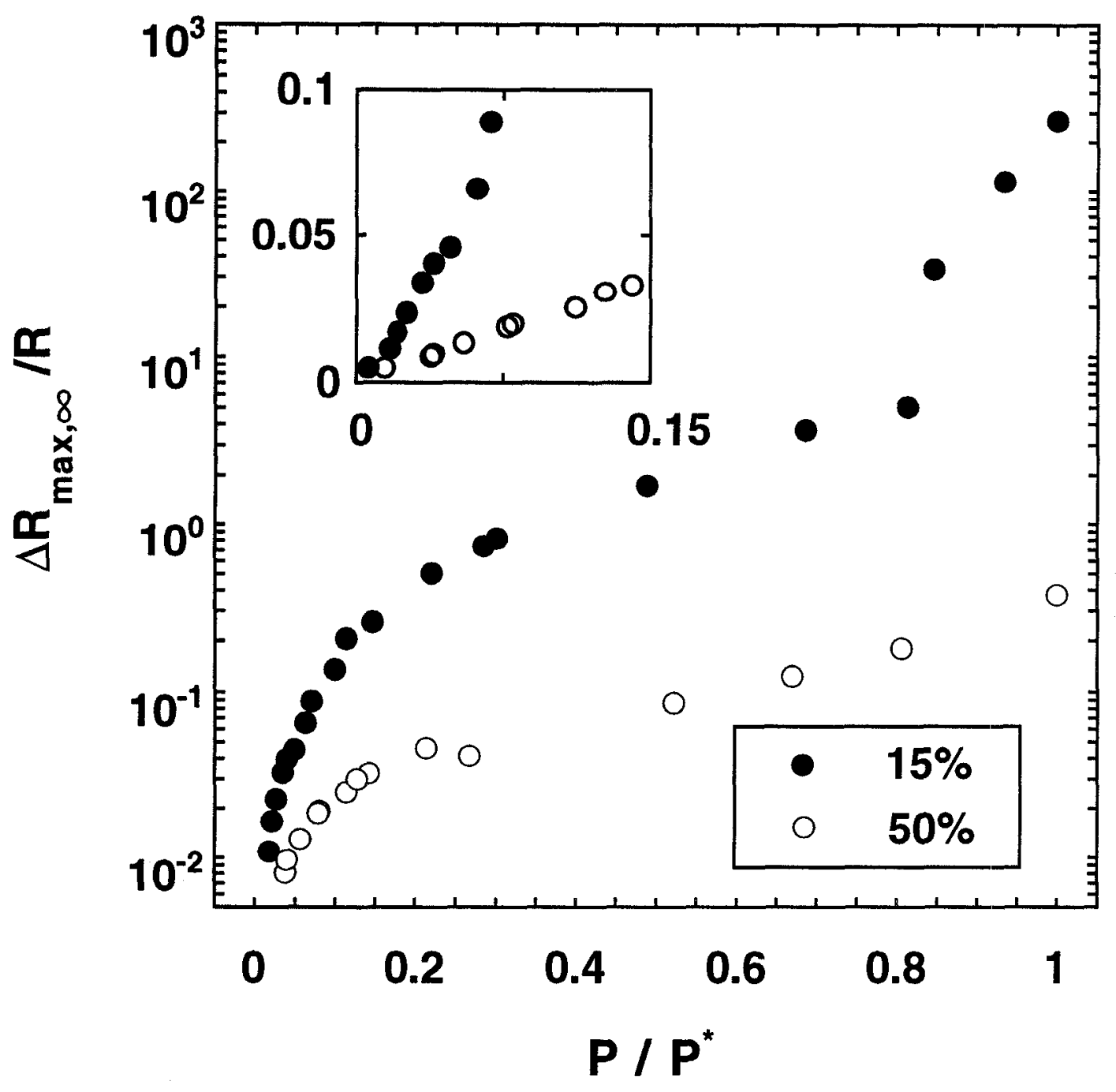

Lonergan et. al., Figure 3 


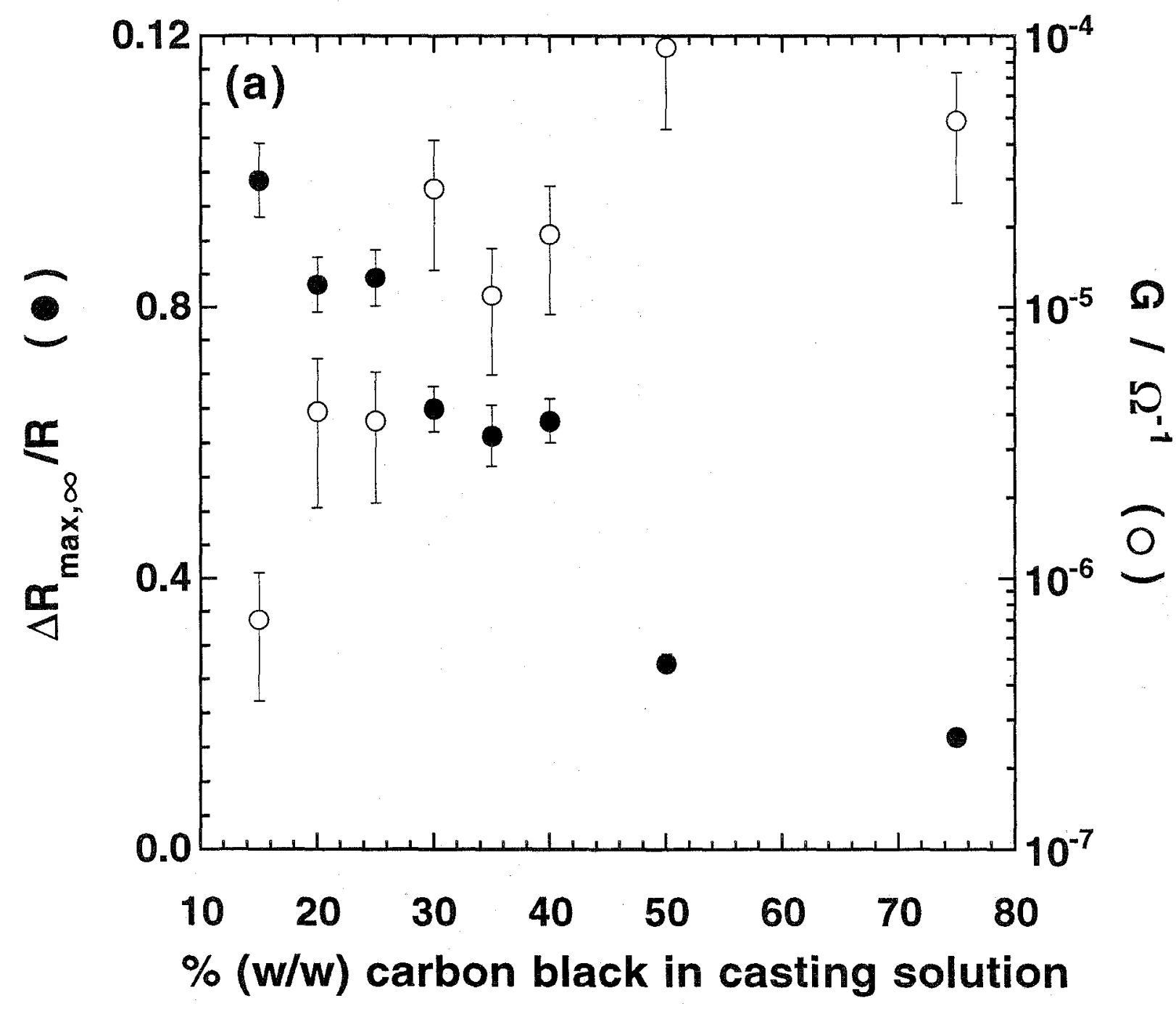

Ionergan et. al., Figure $4 a$ 


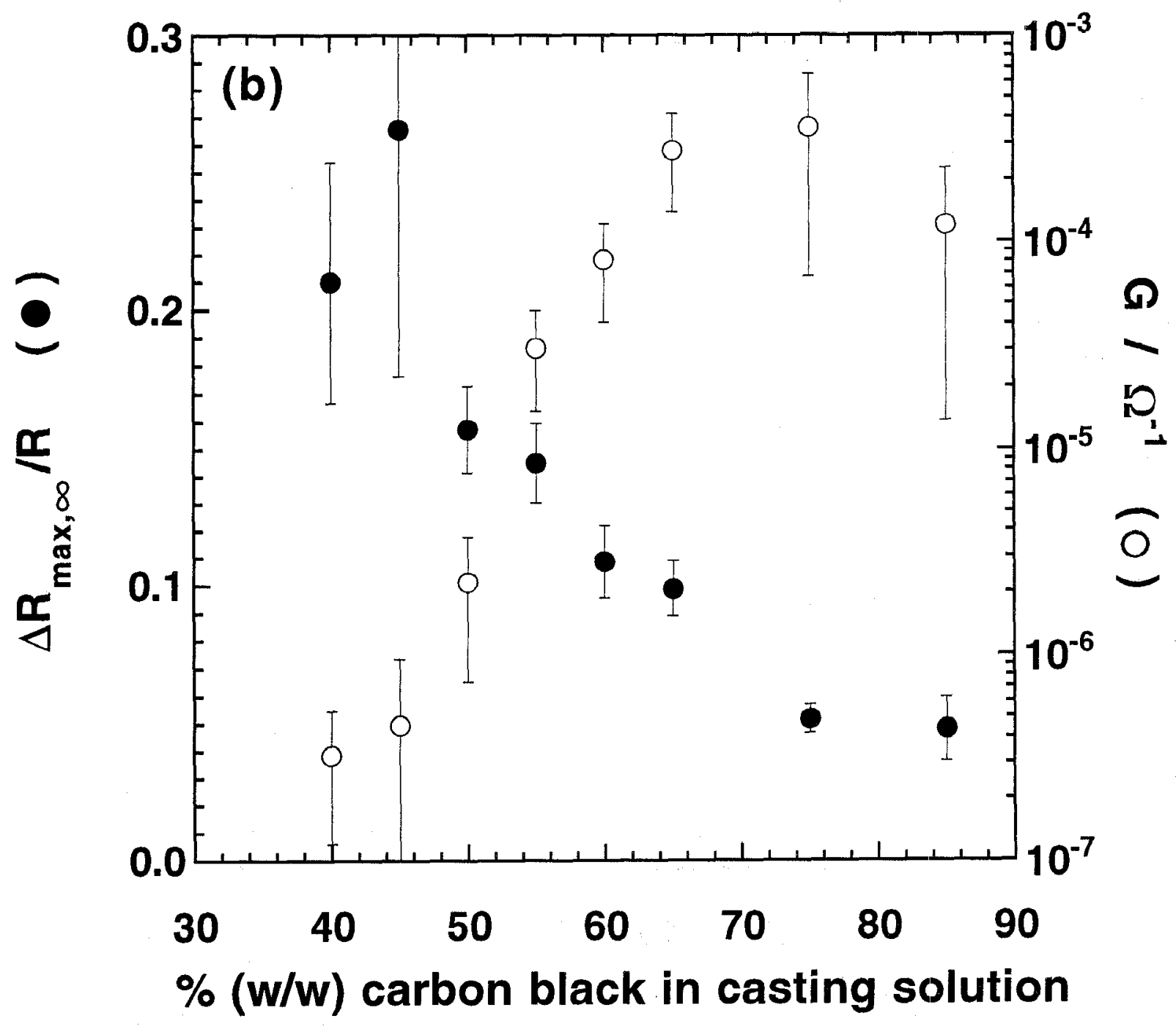

Lonergan et. al., Figure 4b 


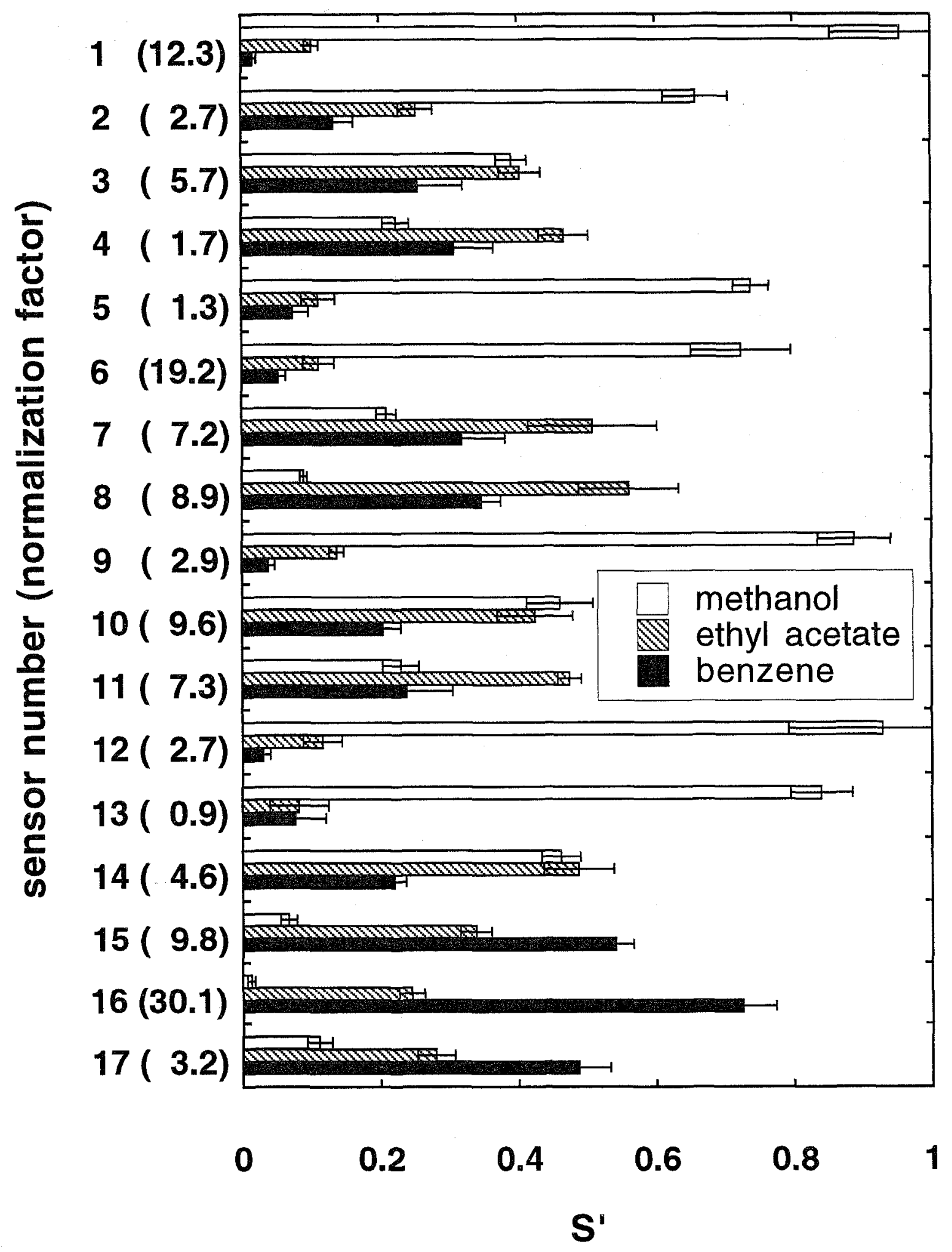

Lonergan et. al., Figure 5 


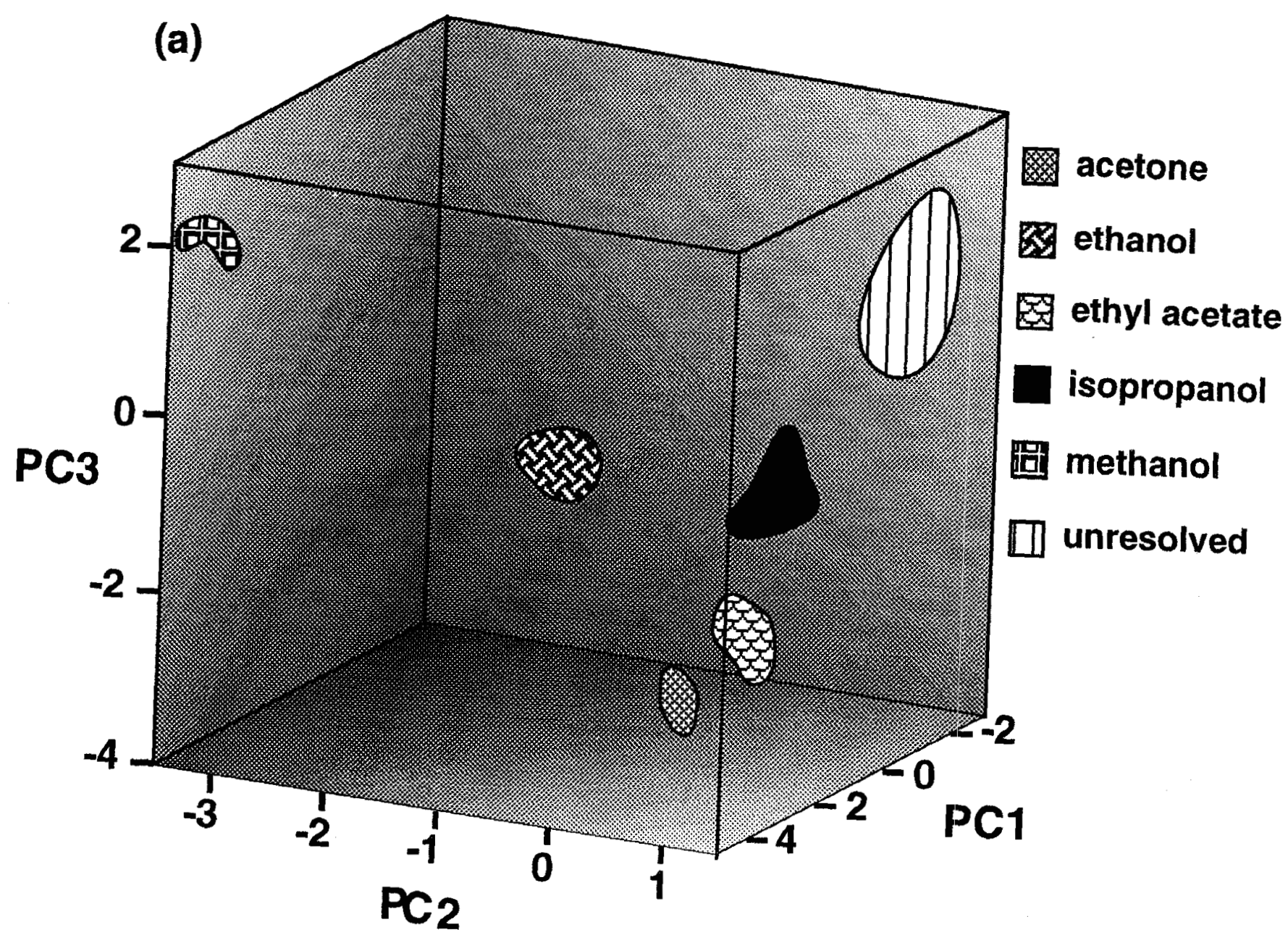

Lonergan et. al., Figure 6a 


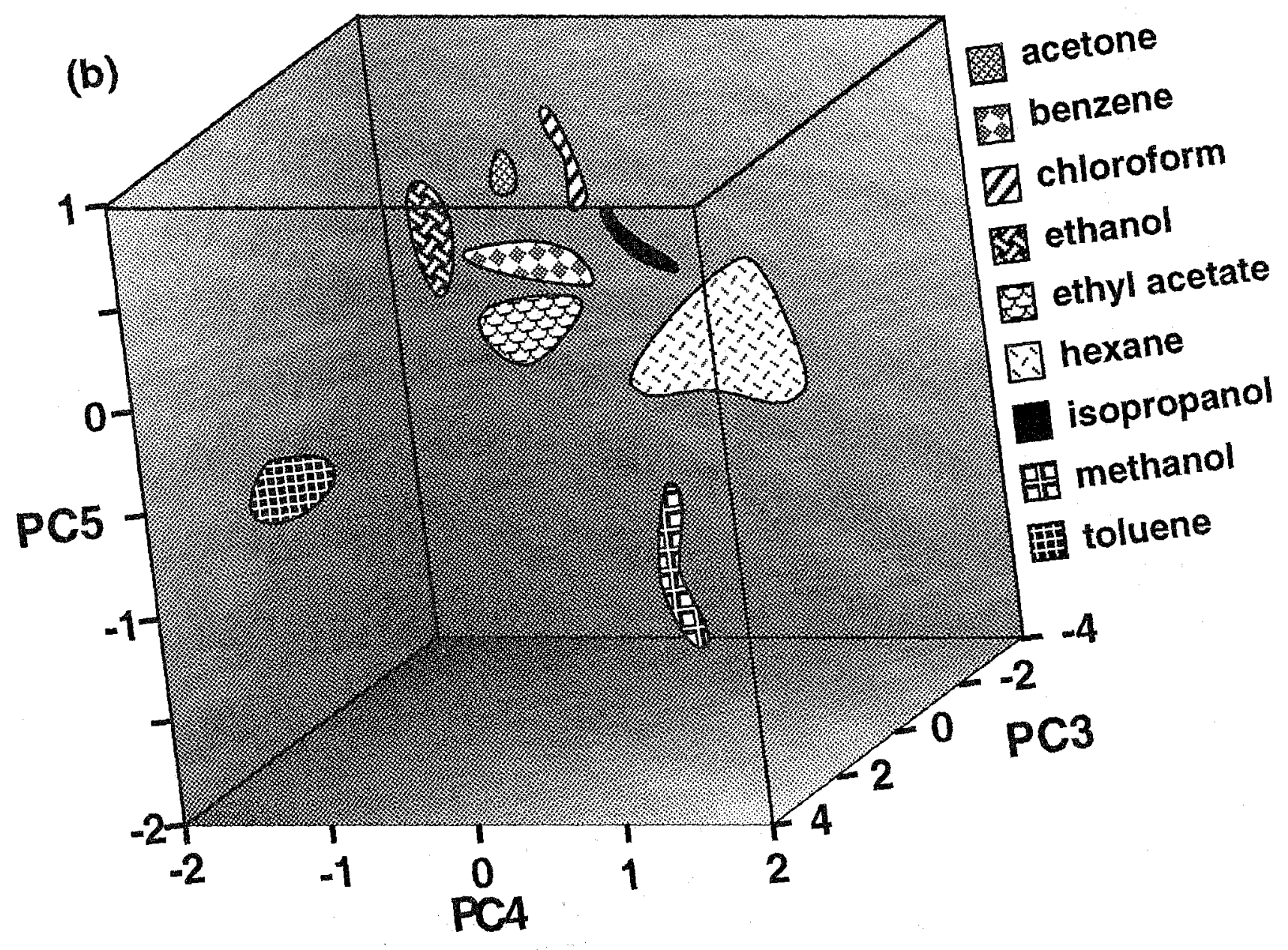

Ionergan et. al., Figure $6 \mathrm{~b}$ 


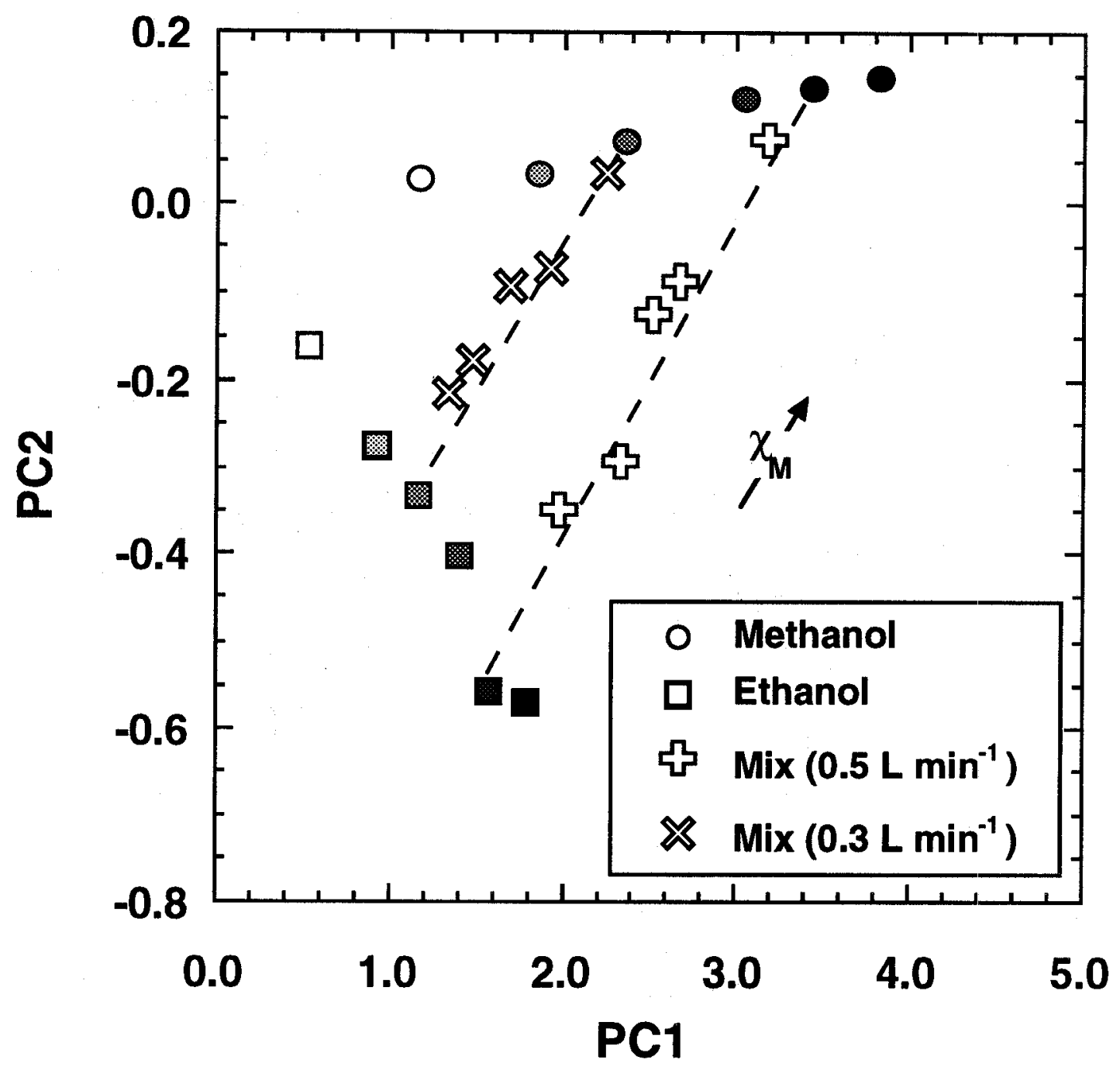

Lonergan et. al., Figure 7 


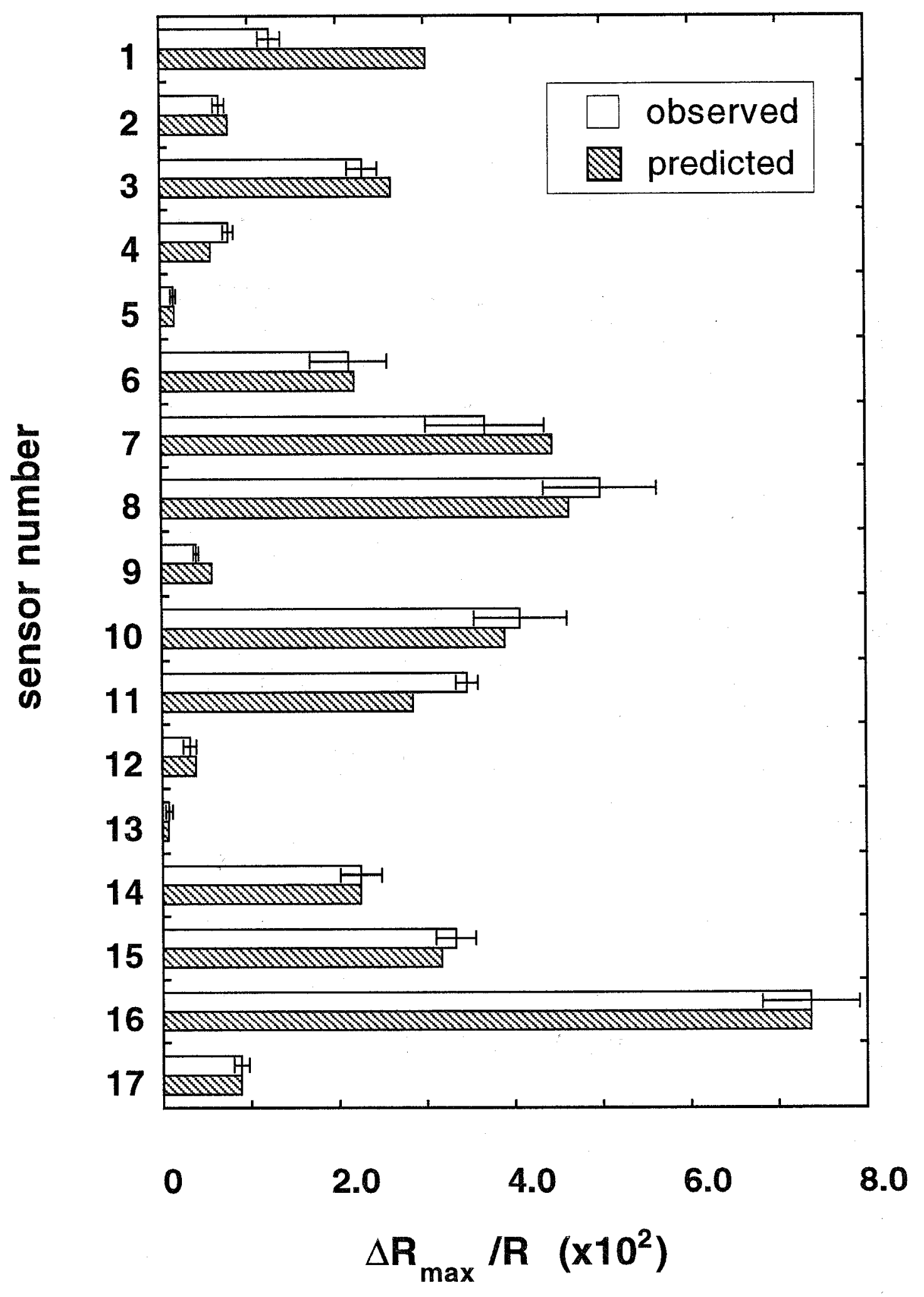

Lonergan et. al., Figure 8 


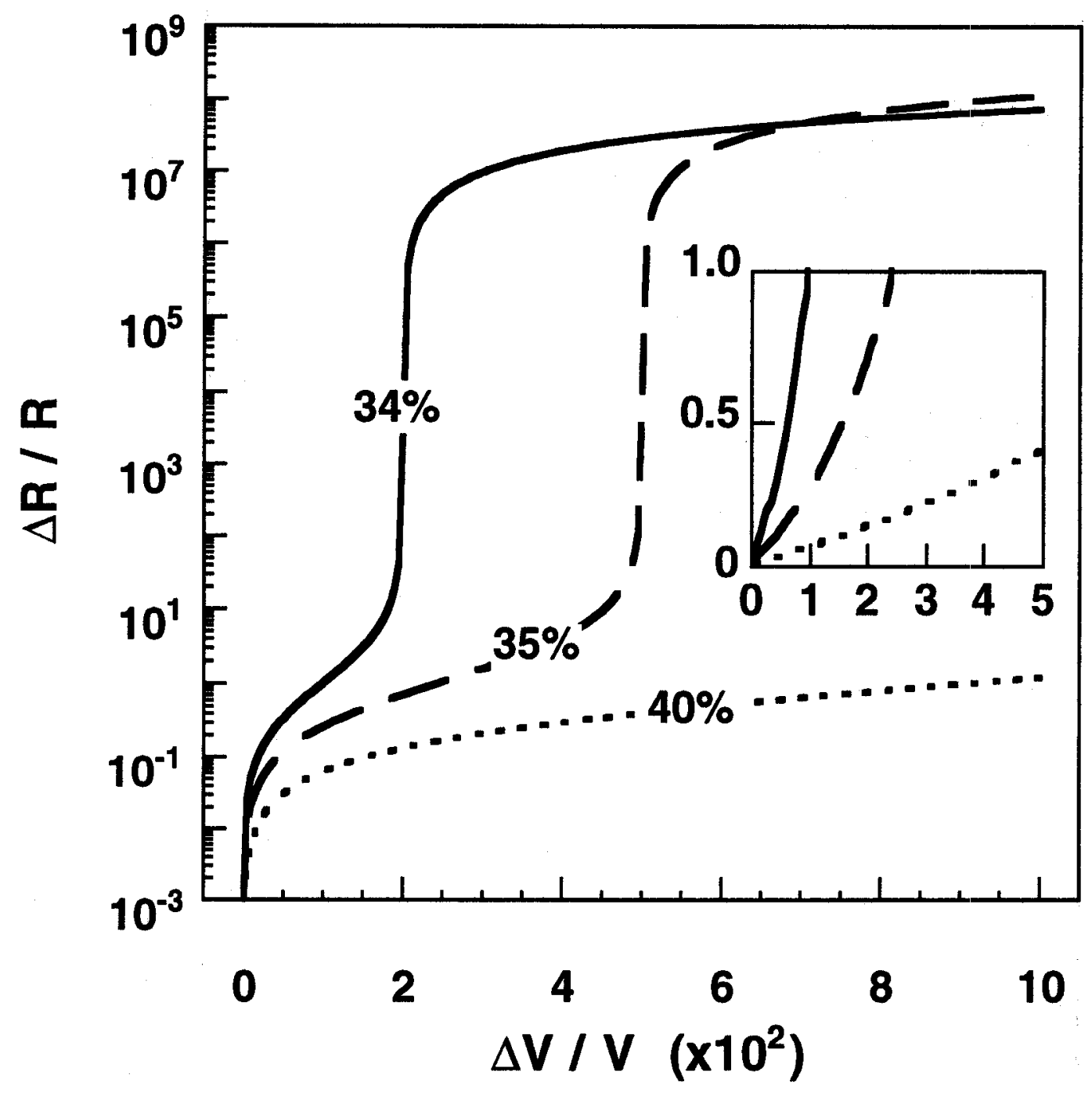

Lonergan et. al., Figure 9 


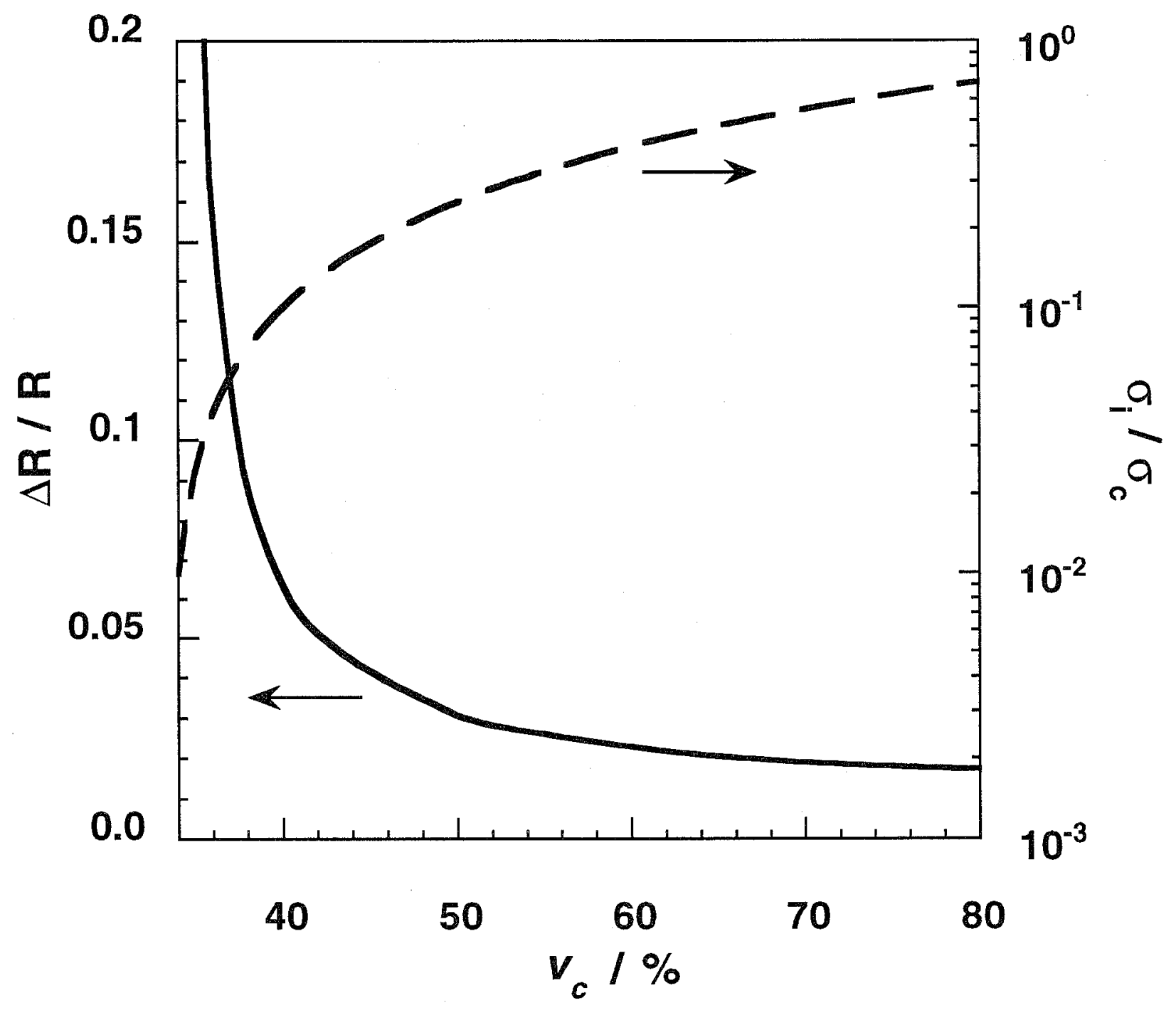

Lonergan et. al., Figure 10 


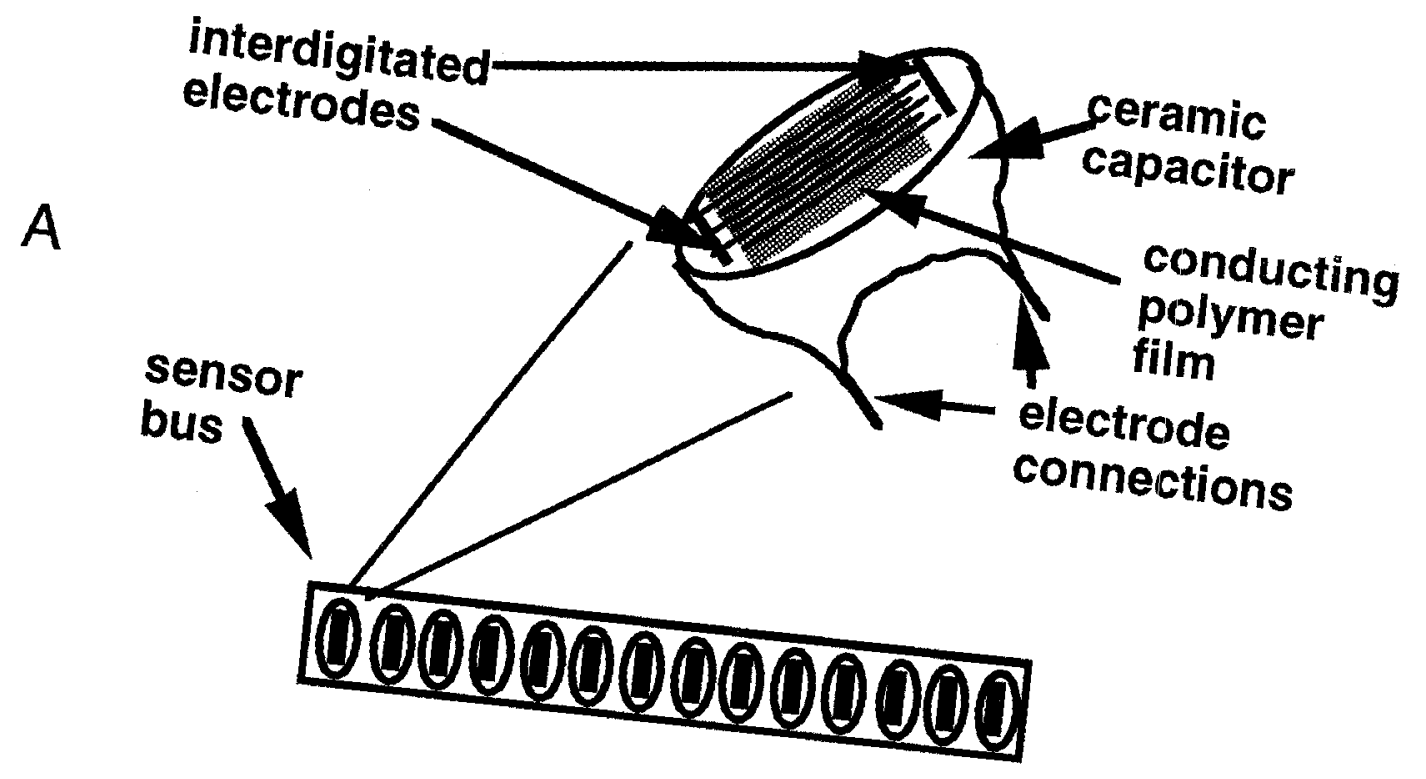

Lonergan et. al., Figure 11a 

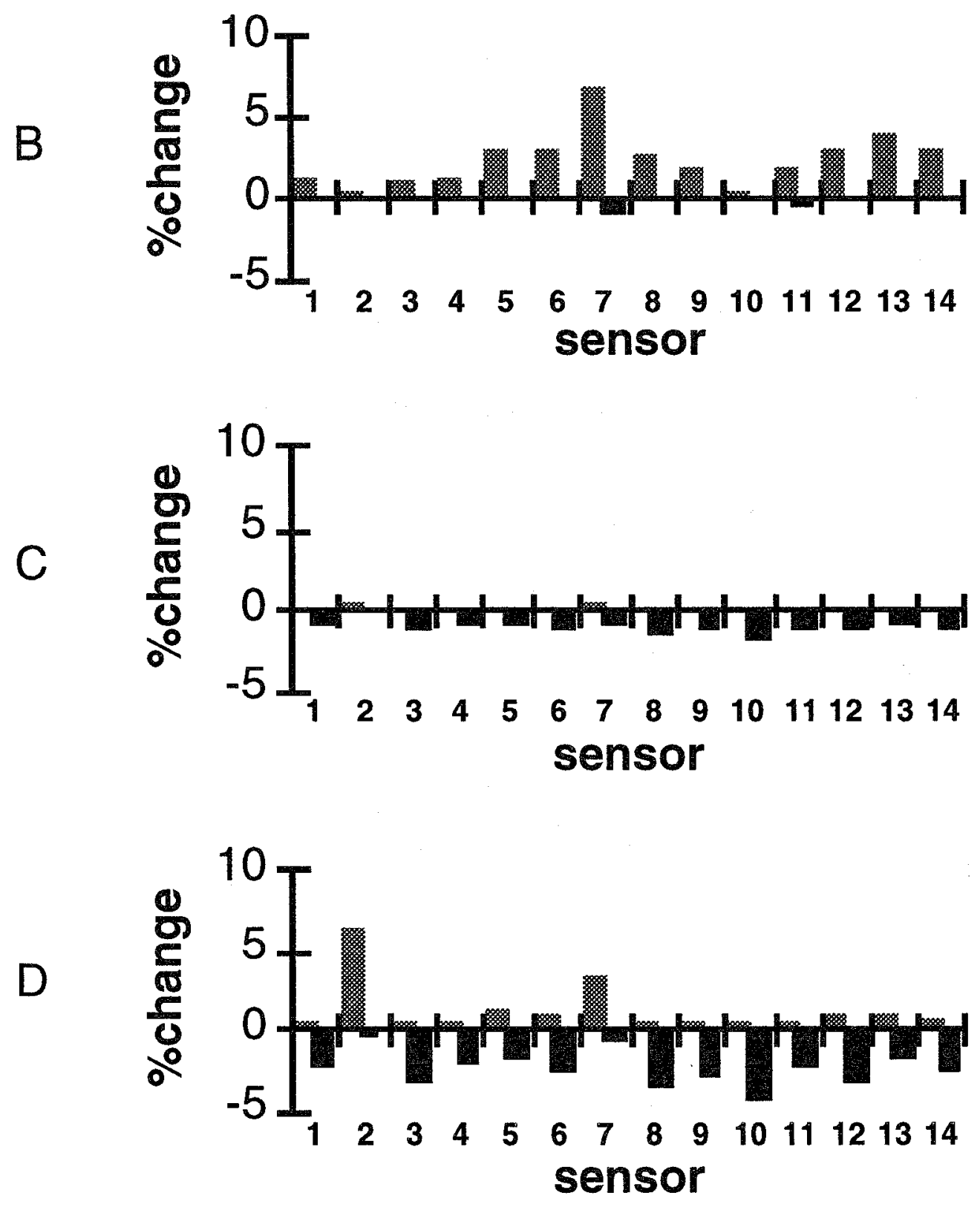

Lonergan et. al., Figure 11 b,c,d 


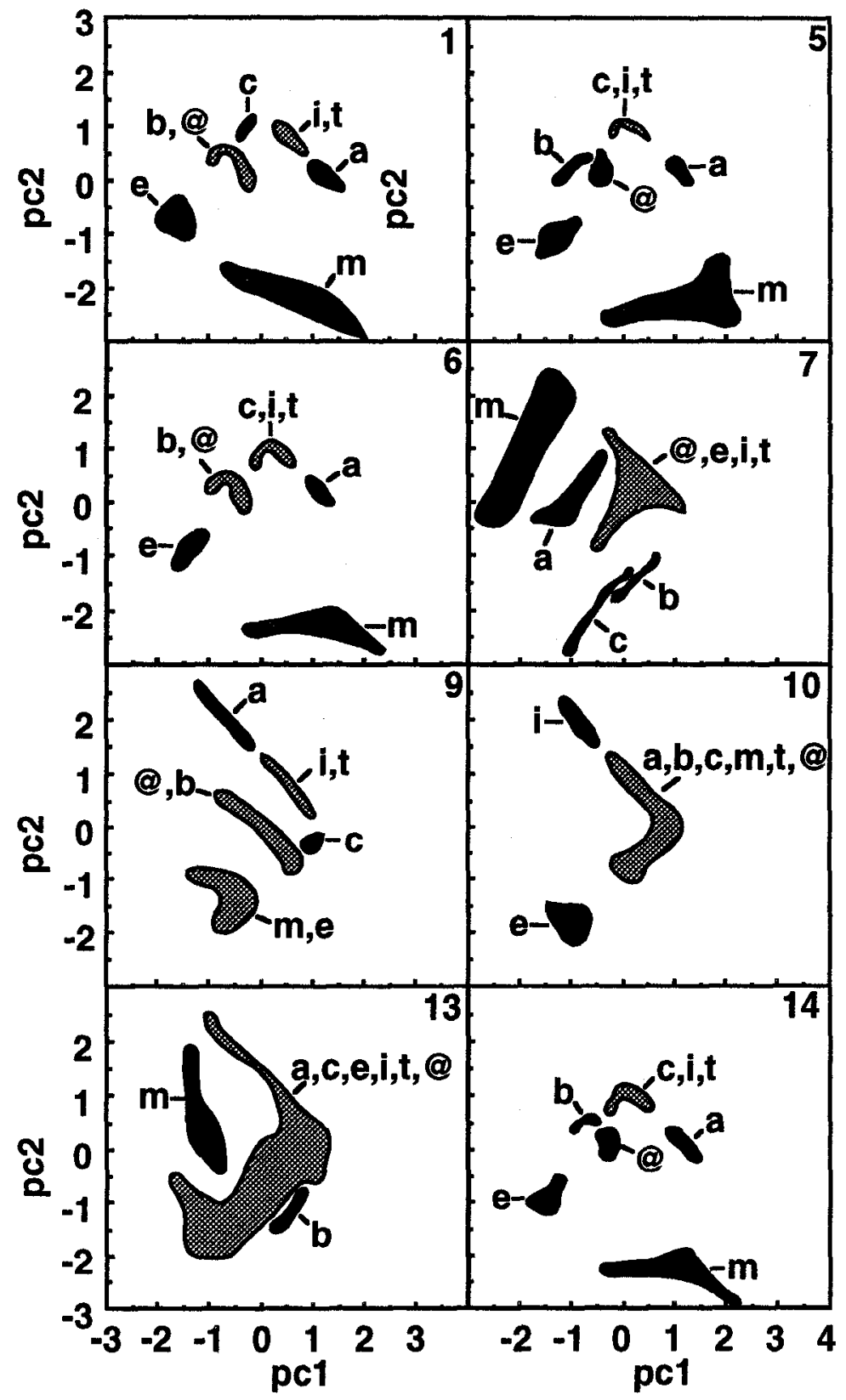

Lonergan et. al., Figure 12 
A

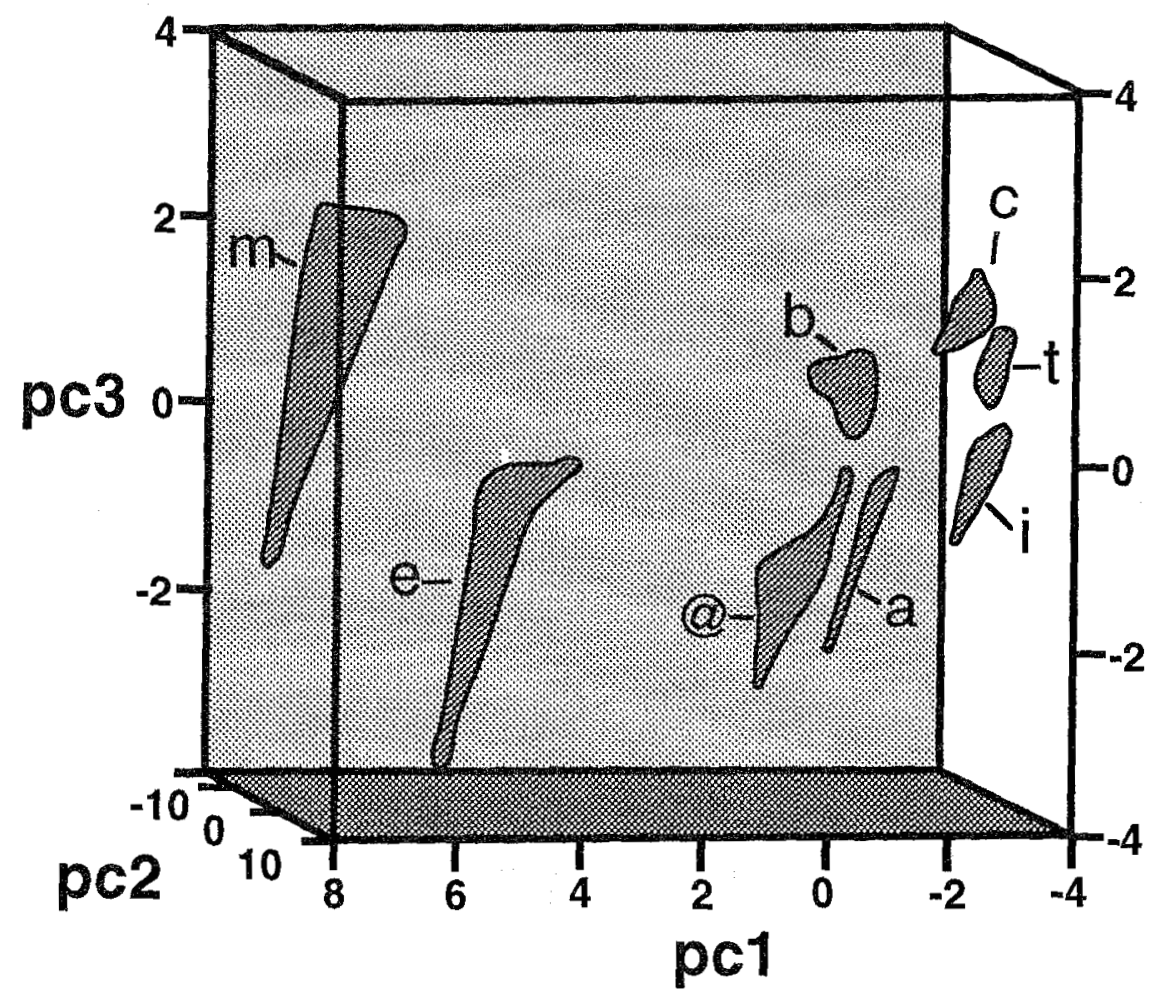

Lonergan et. al., Figure 13a 
B

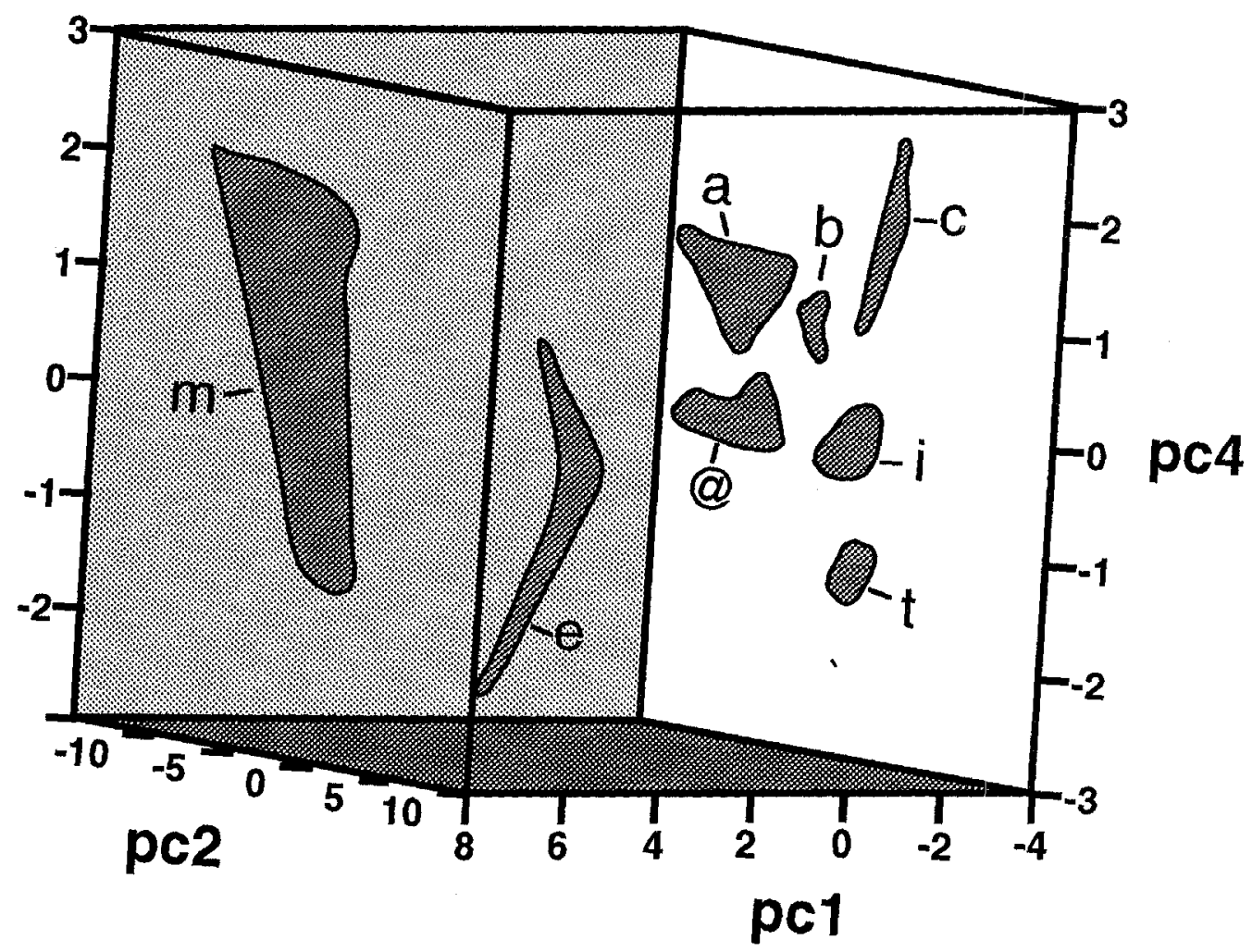

Lonergan et. al., Figure $13 b$ 